PNL-6934

UC-400

\title{
U.S. Department of Energy \\ Radioisotope Customers with \\ Summary of Radioisotope \\ Shipments, FY 1988
}

N. C. Van Houten

June 1989

Prepared for the U.S. Department of Energy under Contract DE-AC06-76RLO 1830

Pacific Northwest Laboratory

Operated for the U.S. Department of Energy

by Battelle Memorial Institute 


\title{
DISCLAIMER
}

This program was prepared as an account of work sponsored by an agency of the United States Government. Neither the United States Government nor any agency thereof, nor Battelle Memorial Institute, nor any of their employees, makes any warranty, express or implted, or assumes any legal liability or responsibility for the accuracy, completeness, or usefulness of any information, apparatus, product, or process disclosed, or represents that its use would not infinge privately owned nghts. Reference herein to any specific commerical product, process, or service by trade name, trademark, manufacturer, or otherwise, does not necessarily constitute or imply its endorsement, recommendation, or favoring by the United States Government or any agency thereof, or Battelle Memorial Institute. The views and opinions of authors expressed herein do not necessarily state or reflect those of the United States Government or any agency thereof.

\section{PACIFIC NORTHWEST LABORATORY operated by \\ BATTELLE MEMORIAL INSTITUTE for the \\ UNITED STATES DEPARTMENT OF ENERGY under Contract DE*ACO6-76RLO 1830}

\author{
Printed in the United States of America \\ Available from \\ National Technical Information Service \\ Unired States Department of Commerce \\ 5285 Port Royal Road \\ Springfield, Virginia 22161 \\ Nris Price Codes \\ Microfiche A01 \\ Printed Copy
}

\begin{tabular}{|c|c|}
\hline & Price \\
\hline Pages & Codes \\
\hline $001-025$ & $\mathrm{~A} 02$ \\
\hline $026-050$ & $\mathrm{~A} 03$ \\
\hline $051-075$ & $\mathrm{AOS}$ \\
\hline $076-100$ & $\mathrm{~A} 05$ \\
\hline $101-125$ & $A 06$ \\
\hline $12 b-150$ & A07 \\
\hline $151-175$ & $A D A$ \\
\hline 176200 & $A D 9$ \\
\hline 201.225 & A 10 \\
\hline $22 \mathrm{~b}-250$ & A 11 \\
\hline 251.275 & A 12 \\
\hline $276-300$ & $\mathrm{~A} 13$ \\
\hline
\end{tabular}


PNL-6934

UC -400

U.S. DEPARTMENT OF ENERGY

RADIOISOTOPE CUSTOMERS

WITH SUMMARY OF

RADIOISOTOPE SHIPMENTS,

FY 1988

N. C. Van Houten

June 1989

Prepared for

the U.S. Department of Energy

under Contract DE-ACO6-76RL0 1830

Pacific Northwest Laboratory

Richland, Washington 99352 



\section{PREFACE}

At the request of the Office of Health and Environment Research (ER-73), Office of Energy Research, U.S. Department of Energy (DOE), Pacific Northwest Laboratory (PNL) annually prepares the radioisotope customer list document. This report covers DOE radioisotope sales and distribution activities by its facilities to domestic, foreign and other DOE facilities for FY 1988 . This is the 25th report in a series dating from 1964 .

The following DOE sites are involved in radioisotope sales and distribution: Argonne National Laboratory (ANL), Brookhaven National Laboratory (BNL), Idaho National Engineering Laboratory (INEL), Los Alamos National Laboratory (LANL), Oak Ridge National Laboratory (ORNL), Pacific Northwest Laboratory (PNL), Savannah River Operations Office (SRO), and Westinghouse Hanford Company (WHC).

The information provided in this report is divided into five sections:

- Radioisotope Suppliers, Facjlity Contacts, and Radioisotopes or Services Supplied. Facilities supplying radioisotopes or services and a contact person for each DOE facility.

- List of Customers, Suppliers, and Radioisotopes Purchased. An alphabetical list of domestic and foreign radioisotope customers and their addresses. Each customer is assigned a customer number that is used to cross-reference data in the other sections of the report. Radioisotope purchases for each customer are listed below their address, along with the supplier code for the laboratory supplying the radioisotopes.

- List of Radioisotopes Purchased Cross-Referenced to Customer Numbers. An alphabetical list of the radioisotopes purchased, cross-referenced with the customer numbers of purchasers.

- Geographic Locations of Radioisotope Customers. An alphabetical list of states and foreign countries cross-referenced with customer numbers of purchasers.

- Radioisotope Sales and Transfers - FY 1988. A table summarizing the FY 1988 radioisotope shipment activities of the DOE laboratory facilities identified in the first section. Radioisotopes are alphabetically listed with the suppliers' abbreviated names. Data on shipments, quantities, units of measure, and dollar values of each radioisotope are listed under domestic, foreign and DOE categories.

The total value of DOE radioisotope sales for FY 1988 was $\$ 11.1$ million. 


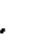




\section{SUMMARY}

Pacific Northwest Laboratory (PNL) prepared this edition of the radioisotope customer list at the request of the Office of Health and Environmental Research (ER-73), Office of Energy Research, U.S. Department of Energy (DOE). This is the 25th report in a serjes dating from 1964. This report covers DOE radioisotope sales and distribution activities by its facilities to domestic, foreign and other DOE facilities for FY 1988.

The report is divided into five sections: radioisotope suppliers, facility contacts, and radioisotopes or services supplied; a list of customers, suppliers, and radioisotopes purchased; a 1 ist of radioisotopes purchased cross-referenced to customer numbers; geographic locations of radioisotope customers; and radioisotope sales and transfers - FY 1988.

Radioisotopes not previously reported in this series of reports were argon-37, arsenic-72, arsenic-73, bismuth-207, gadol inium-151, rhenium-188, rhodium-101, selenium-72, xenon-123 and zirconium-88.

The total value of DOE radioisotope sales for FY 1988 was \$11.1 million, an increase of $3 \%$ from FY 1987. 



\section{ACKNOHLEDGMENTS}

The author would like to thank the U.S. Department of Energy Laboratories and Area offices for their annual data contributions that are used to produce this report:

Argonne National Laboratory Brookhaven National Laboratory Idaho National Engineering Laboratory/EG\&G Idaho, Inc. Los Alamos National Laboratory Oak Ridge National Laboratory Pacific Northwest Laboratory Savannah River Operations Office Westinghouse Hanford Company 



\section{LIST OF PREVIOUS REPORTS}

Previous reports in this series are listed below by report number and fiscal year that radioisotopes were received. All of these reports were published in the year following the year listed (e.g., TID-2160 reports FY 1964 activities, but the report publication date was 1965).

$\begin{array}{lll}\text { TID-2160 (FY 1964) } & \text { BNWL-1774 (FY 1972) } & \text { PNL-3839 (FY 1980) } \\ \text { ORNL-I IC-6 (FY 1965) } & \text { BNWL-1824 (FY 1973) } & \text { PNL-4177 (FY 1981) } \\ \text { ORNL-IIC-9 (FY 1966) } & \text { BNWL-1902 (FY 1974) } & \text { PNL-4759 (FY 1982) } \\ \text { ORNL-I IC-13 (FY 1967) } & \text { BNWL-1993 (FY 1975) } & \text { PNL-5126 (FY 1983) } \\ \text { ORNL-IIC-17 (FY 1968) } & \text { BNWL-2147 (FY 1976) } & \text { PNL-5492 (FY 1984) } \\ \text { ORNL-I IC-27 (FY 1969) } & \text { PNL-2572 (FY 1977) } & \text { PNL-5948 (FY 1985) } \\ \text { ORNL-I IC-32 (FY 1970) } & \text { PNL-2930 (FY 1978) } & \text { PNL-6361 (FY 1986) } \\ \text { ORNL-I IC-38 (FY 1971) } & \text { PNL-3274 (FY 1979) } & \text { PNL-6657 (FY 1987) }\end{array}$

Similar reports covering the U.S. Department of Energy's stable radioisotopes' distribution programs have also been prepared and are available from the National Technical Information Service, U.S. Department of Commerce, 5285 Port Royal Road, Springfield, Virginia 22161 (telephone 703/487-4650).

\section{STABLE ISOTOPE SALES: MOUND FACILITY CUSTOMER AND SHIPMENT SUMMARIES}

Report Number and Date

MLM-2718 (FY 1979)

MLM-2843 (FY 1980)

MLM-2994 (FY 1981)

MLM-3095 (FY 1982)

MLM-3234 (FY 1983)
Report Number and Date

MLM-3366 (FY 1984)

MLM-3468 (FY 1985)

MLM-3494 (FY 1986)

MLM-3550 (FY 1987)

STABLE ISOTOPE CUSTOMER LIST AND SUMMARY OF SHIPMENTS

Report Number and Date

ORNL/TM-7214 (FY 1979)

ORNL/TM-7764 (FY 1980)

ORNL/TM-8302 (FY 1981)

ORNL/TM-8704 (FY 1982)

ORNL/TM-8979 (FY 1983)
Report Number and Date

ORNL/TM-9430 (FY 1984)

ORNL/TM-9866/RI (FY 1985)

ORNL/TM-10324 (FY 1986)

ORNL/TM-10639 (FY 1987)

ORNL/TM-11077 (FY 1988) 


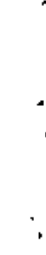




\section{CONTENTS}

PREFACE ................................

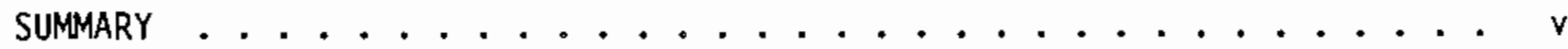

ACKNOWLEDGMENTS ................................. vii LIST OF PREVIOUS REPORTS . .................... . ix LIST OF ABBREVIATIONS ................. xi . . . . .

1.0 RADIOISOTOPE SUPPLIERS, FACILITY CONTACTS, AND RADIOISOTOPES OR SERVICES SUPPLIED ................... 1

2.0 CUSTOMERS, SUPPLIERS, AND RADIOISOTOPES PURCHASED, WITH LISTING OF CUSTOMER NUMBERS ................ 5

3.0 LIST OF RADIOISOTOPES PURCHASED CROSS-REFERENCED TO

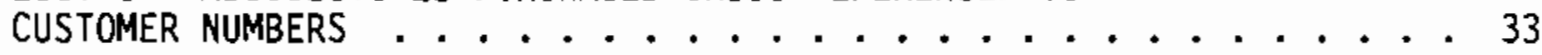

4.0 GEOGRAPHIC LOCATIONS OF RADIOISOTOPE CUSTOMERS . . . . . . . . 39

5.0 RADIOISOTOPE SALES AND TRANSFERS - FY $1988 \ldots . \ldots 43$ 


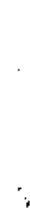




\section{LIST OF ABBREVIATIONS}

\begin{tabular}{|c|c|c|c|}
\hline$A G-105$ & Silver -105 & CE-139 & Cerium-139 \\
\hline AL-26 & Aluminum-26 & $C E-144$ & Cerium-144 \\
\hline AM-241 & Americium-241 & CF -249 & Californium-249 \\
\hline AM-243 & Americium-243 & CF -252 & Cal i forni um-252 \\
\hline AO & $\begin{array}{l}\text { Administration Only; no } \\
\text { shipments made from site }\end{array}$ & $\mathrm{Ci}$ & Curie \\
\hline AR-37 & Argon-37 & $(M-\angle 49$ & curlutil-<4s \\
\hline AS-72 & Arsenic -72 & $C M-248$ & Curium-248 \\
\hline AS -73 & Arsenic-73 & $\mathrm{CO}-57$ & Cobalt-57 \\
\hline AU-195 & Gold-195 & $\mathrm{CO}-58$ & Cobalt-58 \\
\hline$A U-196$ & Gold-196 & $C 0-60$ & Cobalt-60 \\
\hline$A U-198$ & Gold-198 & $\operatorname{cs}-13)$ & Cesium-13/ \\
\hline B-11 & Boron-11 & CSI & $\begin{array}{l}\text { Cyclotron Service } \\
\text { Irradiations }\end{array}$ \\
\hline BA-140 & Barium-140 & CSI-BNL & $\begin{array}{l}\text { Cyclotron Service } \\
\text { Irradiations by Brookhaven }\end{array}$ \\
\hline BI -207 & Bismuth-207 & CSI-LANL & $\begin{array}{l}\text { Cyclotron Service } \\
\text { Irradiations by Los } \\
\text { Alamos }\end{array}$ \\
\hline BK-249 & Berkelium-249 & CU-64 & Copper-64 \\
\hline$B R-77$ & Bromine-77 & & \\
\hline BR-82 & Bromine-82 & $60-65$ & Copper-65 \\
\hline$C-11$ & Carbon-11 & $(0-6)$ & Copper -67 \\
\hline$c-14$ & Carbon-14 & CU-68 & Copper-68 \\
\hline $\mathrm{CA}-47$ & Calcium-47 & DU & Depleted Uranium \\
\hline CAP & Encapsulation & E & Enriched \\
\hline$C D-109$ & Cadmium-109 & $E S-253$ & Einsteinium-253 \\
\hline
\end{tabular}




\begin{tabular}{|c|c|c|c|}
\hline EU & Enriched Uranium & LU-172 & Lutet i um-172 \\
\hline EU-152 & Europi im-152 & LU-173 & Lutet ium-173 \\
\hline$F-18$ & Fluorine-18 & MCP & $\begin{array}{l}\text { Miscellaneous Compound } \\
\text { Preparations }\end{array}$ \\
\hline$F E-52$ & Iron-52 & $\mu \mathrm{Ci}$ & Microcurie \\
\hline $\mathrm{FE}-55$ & Iron-59 & $\mu g$ & Microgram \\
\hline$r[-32$ & Fission Products & nCi & Millicurie \\
\hline$g$ & & mg & Milligram \\
\hline GA-67 & Gallium-67 & MG-28 & Magnes i um-28 \\
\hline GD-148 & Gadol in ium-148 & MI & Miscellaneous Isotopes \\
\hline GD-151 & Gadol inium-151 & MIS & Miscellaneous Quantities \\
\hline GD-153 & Gadol inium- 153 & $\begin{array}{l}\text { MS } \\
M N-54\end{array}$ & $\begin{array}{l}\text { Misce } 1 \text { laneous Services } \\
\text { Manganese-54 }\end{array}$ \\
\hline $\begin{array}{l}\text { GE-68 } \\
\mathrm{H}-3\end{array}$ & $\begin{array}{l}\text { Germanium- } 68 \\
\text { Hydrogen-3 (tritium) }\end{array}$ & $M N-57$ & Manganese -57 \\
\hline$H F-172$ & Hafn i um- 172 & $\mathrm{NA}-22$ & Sodium-22 \\
\hline$H 0-163$ & Holmium-163 & $N A-24$ & Sodium-24 \\
\hline $\mathrm{I}-123$ & Iodine-123 & NB-95 & Niobium-95 \\
\hline$I-125$ & Iodine- 125 & NI -63 & Nickel-63 \\
\hline$I-129$ & Iodine- 129 & NP-237 & Neptunium-237 \\
\hline $1-131$ & Iodine-131 & NP-237H & High Purity NP-237 \\
\hline IN-111 & Indium-111 & NP -2370 & 0xide of NP-237 \\
\hline IR-192 & Iridium-192 & $\mathrm{NU}$ & Natural Uranium \\
\hline $\mathrm{kCi}$ & Kilocurie & OS-191 & 0smium-191 \\
\hline $\mathrm{kg}$ & Kilogram & $\mathrm{P}-32$ & Phosphorus-32 \\
\hline $\mathrm{KR}-85$ & Krypton-85 & $P-33$ & Phosphorus -33 \\
\hline KR-85E & Enriched Krypton- 85 & $\mathrm{PA}-231$ & Protactinium- 231 \\
\hline & & PD-109 & Palladium-109 \\
\hline
\end{tabular}




\begin{tabular}{|c|c|c|c|}
\hline PM-147 & Promethium-147 & SM-151 & Samarium-151 \\
\hline$P 0-208$ & Polonium-208 & SN-117 & Tin-117 \\
\hline$P 0-210$ & Polonium-210 & $S N-119 m$ & Tin-119m \\
\hline PU-236 & P1utoni um-236 & $S P$ & Special Processes \\
\hline PU-237 & Plutonium-237 & SR-82 & St ront i um-82 \\
\hline PU-238 & Plutonium-238 & SR-85 & Strontium-85 \\
\hline PU-239 & Plutonium-239 & SR-89 & Stront ium-89 \\
\hline PU-240 & Plutonium-240 & SR-90 & Stront jum-90 \\
\hline PU-241 & Plutonium-241 & SS & Special Services \\
\hline PU-242 & Plutonium-242 & TB-158 & Terbi ium-158 \\
\hline $\mathrm{RB}-81$ & Rubidium-81 & TC-99 & Technet ium-99 \\
\hline RB-83 & Rubidium-83 & $\mathrm{TE}-128$ & Tellurium-128 \\
\hline RB-86 & Rubidium-86 & TH-229 & Thorium-229 \\
\hline RE -188 & Rhenium-188 & TH-230 & Thorium-230 \\
\hline RH-101 & Rhodium-101 & TH-232 & Thorium-232 \\
\hline RSI & $\begin{array}{l}\text { Reactor Service } \\
\text { Irradiations }\end{array}$ & TH-234 & Thorium-234 \\
\hline $\mathrm{RU}-97$ & Ruthen ium-97 & TI -44 & Titanium- 44 \\
\hline$R U-103$ & Ruthen ium-103 & $T M-167$ & Thul i um-167 \\
\hline RU-106 & Ruthenium-106 & $\mathrm{TM}-170$ & Thul i um-170 \\
\hline RW & $\begin{array}{l}\text { Radioisotopes Withdrawn; } \\
\text { prepared under special } \\
\text { approval }\end{array}$ & $\begin{array}{l}\text { TP } \\
\text { TRU-SV }\end{array}$ & $\begin{array}{l}\text { Target Preparations } \\
\text { Trans Uranium Services }\end{array}$ \\
\hline SE-72 & Selenium-72 & & Technical Service \\
\hline SE-75 & Selenium-75 & $\mathrm{U}-233$ & Uranium-233 \\
\hline SI-31 & Silicon-31 & $U-233 S P$ & $\begin{array}{l}\text { Uranium-233 Special } \\
\text { Services }\end{array}$ \\
\hline$S I-32$ & Silicon-32 & $U-234$ & Uranium-234 \\
\hline SM-145 & Samarium-145 & $\mathrm{U}-235$ & Uranium-235 \\
\hline
\end{tabular}




$\begin{array}{ll}\text { U-236 } & \text { Uranium-236 } \\ \text { U-238 } & \text { Uranium-238 } \\ \text { V-48 } & \text { Vanadium-48 } \\ \text { V-54 } & \text { Vanadium-54 } \\ \text { XE-123 } & \text { Xenon-123 } \\ \text { XE-127 } & \text { Xenon-127 } \\ \text { Y-87 } & \text { Yttrium-87 } \\ \text { Y-88 } & \text { Yttrium-88 } \\ \text { Y-90 } & \text { Yttrium-90 } \\ \text { ZN-65 } & \text { Zinc-65 } \\ \text { ZR-88 } & \text { Zirconium-88 } \\ \text { ZR-95 } & \text { Zirconium-95 }\end{array}$




\subsection{RADIOISOTOPE SUPPLIERS, FACILITY CONTACTS, AND RADIOISOTOPES OR SERVICES SUPPLIED}

This section lists DOE facilities supplying radioisotopes or services, the contact person for each facility, and the radioisotopes or services supplied. If readers need more specific information about radioisotope sales or shipments for a particular facility, they should contact the person listed for that facility. 
RADIOISOTOPE SUPPLIERS, FACILIYY CONTACTS, AND RADIOISOTOPES OR SERVICES SUPPLIED

Radioisotope Supplier

Argonne National Laboratory

Brookhaven National Laboratory
Supplier

Code

ANL J. E. Gindler, Assistant
Director, Chemistry Div. Argonne National Laboratory 9700 South Cass Avenue Argonne, IL 60439

FTS $972-3686$

Commercial (312) 972-3686

BNL K. S. Dahms

Radioisotopes and Special Materials Group

Brookhaven National Laboratory

Upton, Long Is land, NY 11973 FTS 666-4051

Commercial (516) 282-4051

Idaho National Engineering Laboratory
J. E. Brasier

Idaho National Engineering Laboratory

EG\&G ldaho, Inc.

P.0. Box 1625

TRA-628, MS 7101

Idaho Falls, ID 83415

FTS 583-9057

Commercial (208) 526-9057
Radioisotopes or Services

No radioisotopes sold directly. Radioisotopes produced counted in ORNL sales.

AM-241, AR-37, AU-198, BE-7, CM-244, CS-137, CU-64, EU-152, I-123, $\mathrm{I}-125, \mathrm{I}-131, \mathrm{Ml}$, NA-22, P-32, PD-109, RE-188, RSI, RU-97, SM-145, U-235, U-238, $\mathrm{XE}-123$

C0-60, GD-153, IR-192, $\mathrm{P} 0-210, \mathrm{SN}-119 \mathrm{~m}$ 
Radioisotope Supplier

Los Alamos National Laboratory

Los Alamos National Laboratory

Oak Ridge National Laboratory
Supplier

Code.

LANL

Lis

Lisa Schneider

Medical Radioisotope Research

Program

Los Alamos National Lab

Group INC-11, Mail Stop J514

Los A\}amos, NM 87545

FTS $843-4675$

Commercial (505) 667-4675

ORNL

T. A. Danner

1 sotope Distribution Office Oak Ridge National Laboratory

P.0. Box 2008

0ak Ridge, TN 37831-6015

FTS 624-6506

Commercial (615) 574-6506

D. A. Dickman

Safeguards Section

Pacific Northwest Laboratory

P.0. Box 999

Richland, WA 99352

FTS 444-1584

Commercial (509) 376-1584
Radioisotopes or Services

AL-26, AS-72, AS-73, BE-7, BI-207, BR-77, BR-82, CD-109, CU-64, CU-67, GE-68, GD-151 。 NA-22, RB-83, RH-101, SE-72 SR-82, V-48, $X E-127, Y-88 ， Z R-88$

AM-241, AM-243, CAP CE-144, CF-252, CM-244, CM-248, C0-60, CS-137, CSI-BNL, CSI-LANL, EU-152, GO-153, H-3, I-129, IR-192，KR-85, $\mathrm{KR}-85 \mathrm{E}, \mathrm{MCP}, \mathrm{MG}-28$, MS, NP-237H, PU-237, PU-238, $P U-239, \quad P U-242$, RSI, RU-106, SP, SR-90, SS, TC-99, TH-229,

TH-230, TP, TRU-SV, TS, $\mathrm{U}-233, \mathrm{U}-234, \mathrm{U}-235$, $\mathrm{U}-236, \mathrm{U}-238, \mathrm{XE}-127$. $\mathrm{Y}-90$

No radioisotopes produced in FY 1988 
Radioisotope Supplier

Savannah River Operations office

Westinghouse Hanford Company
Supplier

Code

SR0

R. L. Chandler, 0irector Processing Division

U.S. Department of Energy

Savannah River Operations Office

P.0. Box A

Aiken, SC 29802

FTS 239-5530

Commercial (803) 725-5530

WHC

R. W. Powe11, Manager Engineering Development West inghouse Hanford Company P.0. 80x 1970

Richland, WA 99352

FTS 444-4529

Commercial (509) 376-4529
Radioisotopes

or Services

No radioisotopes sold

directly. Radio-

isotopes produced

counted in ORNL sales.

No radioisotopes sold

directly. Radio-

isotopes produced

counted in ORNL sales. 
2.0 CUSTOMERS, SUPPLIERS, AND RADIOISOTOPES PURCHASED, WITH LISTING OF CUSTOMER NUMBERS

This section is an alphabetical listing of domestic and foreign radioisotope customers and their addresses. Each customer has a customer number that is used to cross-reference data in Sections 3.0 and 4.0 of this report. Radioisotope purchases for each customer are listed below each address, along with the supplier code for the laboratory supplying the radioisotopes. 
ALPHABETICAL LISTING (BY COUNTRY) OF CUSTOMERS, SUPPLIERS, AND ISOTOPES PURCHASED

\section{UNITED STATES}

1 AT\&T Bell Laboratories

P.0. Box 800

Short Hills, NJ 07078

ORNL: TP, U-235

2 AT\&T Technologies

P.0. Box 105229

Atlanta, GA 30348

ORNL: KR-85

3 Abbott Laboratories

P.0. Box 177

Abbott Park, IL 60064

ORNL: $\quad Y-90$

4 Accu Ray Corporation

650 Ackerman Road

P.0. Box 00248

Columbus, OH 43202

ORNL: KR-85

5 Advanced Micro Devices, Inc.

P.0. Box 3453, M/S 13

Sunnyvale, CA 94088-3453

ORNL: KR-85

6 Aeonic Systems, Inc. 46 Manning Road

Billerica, MA 01821

ORNL: $\quad K R-85$
7 Agnes Scott College

E. College Avenue

Decatur, GA 30030

ORNL: TC-99

8 Albert Einstein College of Medicine

1300 Morris Park Avenue

Bronx, NY 10461

LANL: CU-67

ORNL: CSI

9 Amersham Corporation 40 North Avenue

Burlington, MA 01803

ORNL: IR-192

10 Argonne National Laboratory 9700 South Cass Avenue Argonne, IL 60439

BNL: RSI

LANL: ZR-88

ORNL: CF-252, CSI, MIS, SP, TH-230, TP, U-233, $\mathrm{U}-234$

11 Argonne National Laboratory P.0. Box 2528

Idaho Falls, ID 83401

ORNL: KR-85, TRU-SV

12 Arizona State University

Tempe, AZ 85287

ORNL: SP, TH-229 
13 Atlantic Richfield Hanford Co.

P.0. Box 769

Richland, WA 99352

ORNL: PU-236, RSI

14 Atom Sciences, Inc.

114 Ridgeway Center

oak Ridge, TN 37830

LANL: $\quad A L-26$

ORNL: CSI

15 Babcock \& Wilcox Company

Naval Nuclear Fuel Division Lynchburg, VA 24505

BNL : $\quad U-235$

16 Bell Telephone Laboratories, Inc.

600 Mountain Avenue

Murray Hill, NJ 07974

BNL: $\quad U-238$

17 Bendix Corporation, The 2000 East Bannister Road

Kansas City, M0 64131

ORNL: $\quad$ SS

18 Beth Israel Hospital

330 Brookline Avenue

Boston, MA 02115

ORNL: $M C P$

19 Bethlehem Steel Corporation

Bldg. G, Room 157

Bethlehem, PA 18016

BNL: $\quad$ RSI
20 Bionetics Research, Inc. 1330-A Piccard Drive Rockville, MD 20850

ORNL: $Y-90$

21 Biosources, Limited

4-4 Bud Way

Nashua, NH 03063

ORNL: GD-153

22 Boston College

Chestnut Hill, MA 02167

ORNL： TC-99

23 Brigham \& Womens Hospital HMS-PBBH-SFCC

Radiology Foundation, Inc.

75 Francis Street

Boston, MA 02115

ORNL: GD-153

24 Brookhaven National Laboratory

Isotope \& Special Materials Group

Upton, Long Is land, NY 11973

LANL: GE-68

ORNL: CSI

25 California Institute of Technology

Pasadena, CA 91125

ORNL: CSI

26 California State University 800 North State College Blvd.

Fullerton, CA 92634

LANL: $\quad$ CU-67

ORNL: CSI, GD-153 
27 Calm VA-1-(525)

P.0. Box 2971

Austin, TX 78769

ORNL: XE-127

28 Canberra Industries, Inc. One State Street

Meriden, CT 06450

ORNL: $\quad$ I-129

29 Case Western Reserve University

2220 Circle Drive

Cleveland, $\mathrm{OH} \quad 44106$

ORNL: $\quad M G-28$

30 Center for Molecular Medicine and Immunology

1 Bruce Street

Newark, NJ 07103

LANL: $Y-88$

ORNL: CSI, Y-90

31 Centocor

244 Great Valley Parkway

Malvern, PA 19355

ORNL: $\quad$ TC-99

32 Children's Hospital

300 Longwood Avenue

Boston, MA 02115

BNL: RSI

ORNL: TC-99

33 Columbia University

168th and Fort Washington Ave.

New York, NY 10032

BNL: PD-109
34 Computer Technology and Imaging, Inc.

810 Innovation Drive Knoxville, TN 37932

LANL: GE-68

ORNL: CSI

35 Consolidated Controls Corp. P.0. Box 726

Springfield, VA 22150

ORNL: $H-3, S S$

36 Cornell University

120 Maple Avenue

Ithica, NY 14850

ORNL: CSI, MG-28

37 Coulter Immunology

440 West 20th St.

Hialeah, FL 33010-2426

LANL: $Y-88$

ORNL: CSI, Y-90

38 Cryogenic Rare Gas Laboratories, Inc. 913 Commerce Circle Hanahan, SC 29410

ORNL: KR-85

39 Cytogen Corporation 201 College Road East

Princeton, NJ 08540

LANL: $\quad Y-88$

ORNL: CSI, SS, Y-90

40 Digital Equipment Corp. 100 Minuteman Road Andover, MA 01810

ORNL: $\quad K R-85$ 
41 Dow Chemical Company

P.0. Box 191

Midland, MI 4864D

ORNL: $\quad Y-90$

42 Dow Chemical USA

Texas Operations

Freeport, TX 77541

ORNL: $\quad Y-90$

43 Downstate Medical Center 450 Clarkson Avenue

Brooklyn, NY 11203

BNL: I-125

44 Drexel University 32nd and Chestnut Streets Philadelphia, PA 19104

ORNL: SS

45 Duke University

P.0. Box 40005

Durham, NC 27706

ORNL: CSI

46 Duke University Medical Center Bell Building, Room 133

Durhan, NC 27710

LANL: GE-68

47 E. I. DuPont de Nemours and Company, Inc.

New England Nuclear Products

549 Albany Street

Boston, MA 02118

ORNL: CSI, GD-153, H-3, I-129, KR-85， SR-90， TC-99, $Y-90$
48 E. I. DuPont de Nemours and Company, Inc. Savannah River Plant Aiken, SC 29801

ORNL: SS

49 E. I. DuPont de Nemours and Company, Inc. Experimental Station Wilmington, DE 19898

ORNL: TC-99

50 E. I. DuPont de Nemours and Company, Inc.

New England Nuclear Products

331 Treble Cove Road

North Billerica, MA 01862

INEL: $\quad$ SN-119m

LANL: GE-68, NA-22, Y-88

51 E. R. Squibb \& Sons

P.0. Box CN 5303

Princeton, NJ 08540

ORNL: CSI， GD-153，XE-127

52 E. R. Squibb and Sons, Inc. Route 1 South, Gate 125

New Brunswick, NJ 08902

LANL: SR-82, Y-88

53 EAL Corporation 2030 Wright Avenue Richmond, CA 94804

ORNL: RSI 
54 EG\&G Idaho, Inc.

INEL Site

P.0. Box 1625

Idaho Falls, ID 83415

BNL: AU-198, RSI

ORNL: CF-252, MIS

55 Eagle-Picher Industries, Inc. Chemsyn Science Laboratories 13605 West 96th Terrace Lenexa, KS 66215

ORNL: $\mathrm{H}-3$

56 Eastman Kodak Company Kodak Park Division

66 Eastman Avenue

Rochester, NY 14650

LANL: RH-101

ORNL: CSI

57 Eberline/TMA

5635 Kircher Blvd., N.E.

Albuquerque, NM 87109

BNL: $\quad A M-241$

58 Environmental Engineering and Testing

P.0. Box 1012

Richland, WA 99352

ORNL: KR-85

59 Fairchild Camera \& Instrument Corporation

Linear Division

P.0. Drawer 7282

Mt. View, CA 94042

ORNL: KR-85
60 Fairchild Semiconductor Corp. P.0. Box 5000, M/S 2C07

Puyallup, WA 98373-0900

ORNL: KR-85

61 Fermi National Accelerator Laboratory

Batavia, IL 60510

BNL: U-238

62 Florida State University

Tallahassee, FL 32306

ORNL: MIS, SP, TC-99, TH-230

63 Ford Aerospace and Communications Corp. Aeronautic Division Ford Road

Newport Beach, CA 92660

ORNL： KR-85

64 Frontier Technology Corp. P.0. Box 486

1641 Burnett Drive

Xenia, OH 45385

ORNL: CF-252, MIS, TRU-SV

65 GA Technologies, Inc.

P.0. Box 85608

San Diego, CA 92138

ORNL: H-3，SR-90，SS

66 GTE Products Corporation Hawes Street

Towanda, PA 18848-0504

LANL: $\quad$ AS-73

ORNL: CSI 
67 Gamma Industries, Inc. 2255 Ted Dunham Avenue Baton Rouge, LA 70802

ORNL: IR-192

68 Gearhart Industries

P.0. Box 1936

Fort Worth, TX 76101

ORNL: $\mathrm{H}-3$

69 General Electric Company Lynchburg, VA 24502

ORNL: KR-85

70 General Electric Company P.0. Box 254

San Jose, CA 95103

ORNL: CF-252, MIS

71 General Electric Company Research and Development Building $\mathrm{K}-1$

Schenectady, NY 12301

BNL: RSI

72 General Electric/SCO 230 E. Goddard Blyd. King of Prussia, PA 19406

BNL: RSI

73 General Semiconductor, Inc. 2001 West 10th Place Tempe, AZ 85281

ORNL: KR-85
74 Genicom Corporation Genicom Drive Waynesboro, VA 22980-1999

ORNL: $\mathrm{KR}-85$

75 Georgia Institute of Technology 225 North Avenue, N.W. Atlanta, GA 30332

ORNL: KR-85

76 Gollob Analytical Service 47 Industrial Road Berkeley Heights, NJ 07922

ORNL: $\mathrm{H}-3, \mathrm{KR}-85$

77 Grumman Aerospace Corporation Research Department South Oyster Bay Road Bethpage, NY 11714

BNL : RSI

78 Harris Semiconductor P.0. Box 883 Melbourne, FL 32901

ORNL: KR-85

79 Hughes Aircraft Company Hughes Microelectronics Center P.0. Box H, 500 Superior Ave. Newport Beach, CA 92658-8903

ORNL: $\quad K R-85$

80 Humanoid Systems

17022 Montanero Street

Carson, CA 90746

ORNL: PU-238, TP 
81 Hunter College

Department of Chemistry

126 E. 69th Street

New York, NY 10021

LANL: $\quad$ CU -67

82 Hybritech Incorporated

P.0. Box 269006

San 0iego, CA 92121

ORNL: $\quad Y-90$

83 IBM Corporation

P.0. Box 9452

New Haven, CT 06534-0452

ORNL: CSI, RSI

84 IBM Research Center

Route 134

Yorktown, NY 10598

LANL: $\quad$ CU-67

85 ICN Biomedicals, Inc.

P.0. Box 19536

Irvine, CA 92714

ORNL: $\quad$ CS $-137, H-3$, MCP

86 IT Corporation

312 Directors Drive

Knoxville, TN 37923

ORNL: SP, U-235

87 Indiana University

P.0. Box 4040

Bloomington, IN 47402

ORNL: $M G-28$
88 Indiana University Northwest

3400 Broadway

Gary, IN 46408

ORNL: $M G-28$

89 Industrial Nuclear Company 1428 Park Avenue

Hoboken, NJ 07030

ORNL: IR-192

90 Industrial Nuclear Company 2506 Davis Street

San Leandro, CA 94577

ORNL: IR-192

91 Iotech, Inc.

11080 Ima Drive

Korthglenn, CO 80233

ORNL: $\quad C 0-60$

92 Iowa State University

Ames Laboratory

Ames, IA 50011

ORNL: $\quad$ TC-99

93 Isotope Products Lab 1800 N. Keystone St. Burbank, CA 91504

LANL: BE-7, CD-109, GD-151, GE-68, XE-127, Y-88

94 Isotope Products Laboratories 404 South Luke Street

Burbank, CA 91502

ORNL: AM-241, CAP, CE-144, CSI, GD-153, I-129, KR-85, PU-239, RU-106, SP, SS, XE-127 
95 Isovac Engineering, Inc. 932 Grand Central Ave. Glendale, CA 91201

ORNL: KR-85

96 J. L. Shepherd and Associates 1010 Arroyo Street

San Fernando, CA 91340

ORNL: CS-137, MCP, RSI

97 John Muir Cancer and Aging Research Institute 2055 N. Broadway Walnut Creek, CA 94596

LANL: $Y-88$

ORNL: CSI, Y-90

98 Johnson Matthey, Inc. P.0. Box 733

Valley Forge, PA 19482-0733

ORNL： TC -99

99 Josiyn Electronic Systems P.0. Box 817 Goleta, CA 93116

ORNL: $\quad H-3$

100 Kaman Instrumentation Corp. P.0. Box 7463

Colorado Springs, c0 80933

ORNL: RSI

101 LSI Logic Corporation 1551 McCarthy Boulevard Milpitas, CA 95035

ORNL: KR-85
102 Lawrence Berkeley Laboratory One Cyclotron Road Berkeley, CA 94720

LANL: BR-77; SR-82

ORNL: CSI, H-3, PU-242, SP, TP

103 Lawrence Livermore National Laboratory

Box 808

Livermore, CA 94550

LANL: RB-83

ORNL: AM-241, CAP, CF-252, CSI, MIS, PU-242, SP, TP

104 Lockheed Missiles \& Space Co. Product Assessment Plant B1dg. 150 - Column D7 Sunnyvale, CA 94088

BNL: NA-22

105 Los Alamos National Laboratory P.0. Box 1663 Los Alamos, NM 87545

BNL: NA-22, RSI

LANL: BR-82, CD-109, CD-109, CU $-64, \mathrm{CU}-67, \mathrm{SE}-72$

ORNL: CM-244, CSI, H-3, I-129, MCP, PU-237, SP, SS, TP, U-233, U-236

106 Lovelace Biomedical \& Environmental Research Institute Bldg. 9217, Area $Y$ Kirtland Air Force Base East Albuquerque, NM 87115

BNL: $\quad B E-7$

LANL: $B E-7$

ORNL: CSI, PU-242, SP 
107 M. D. Anderson Hospital

151 Holcombe

Houston, TX 77030

LANL: GE-68

108 Mallinckrodt, Inc.

2703 Wagner Place

Maryland Heights, M0 63043

ORNL: CSI, XE-127

109 Manufacturing Sciences Corp. 804 Kerr Hollow Road

Oak Ridge, TN 37831

BNL: $U-238$

110 Marine Biological Laboratory Wood Hole, MA 02543

ORNL: MG-28

111 Martin Marietta Corporation East Sand Lake Road Orlando, FL 32855

ORNL： KR-85

112 Martin Marietta Energy Systems Oak Ridge Gaseous Diffusion Plant

P.0. Box P

Oak Ridge, TN 37830

ORNL: KR-85, TP, U-235

113 Martin Marietta Energy Systems Oak Ridge National Laboratory Isotope Distribution Office P.0. Box X

Oak Ridge, TN 37831-6615

INEL: GD-153，IR-192
114 Martin Marietta Energy Systems, I

Y-12 Plant

P.0. Box $Y$

Oak Ridge, TN 37831

ORNL: GD-153, IR-192, RSI, SR-90, SS

115 Massachusetts Institute of Technology

96 Prescott Street

Cambridge, MA 02139

BNL: $\quad$ CU-64

116 Measurex Corporation 10475 Imperial Avenue Cupertino, CA 95014

ORNL: CE-144, KR-85

117 Medical \& Scientific Enterprises Inc. 56 Union Avenue Sudbury, MA 01776

ORNL: GD-153

118 Medical Research Foundation 181-A Upper Riverdale Road Riverdale, GA 30274

ORNL: SS, Y-90

119 Micra Corporation 120 Ricefield Lane Hauppauge, NY 11788

ORNL: KR-85

120 Micro-Rel, Inc. 2343 W. 10th Place Tempe, AZ 85281

ORNL: KR-85 
121 Minnesota Mining and

Manufacturing $\mathrm{Co}$.

P.0. Box 33121

St. Pau 1, MN 55133

ORNL： GD-153，KR-85

122 Moravek Biochemicals, Inc.

577 Mercury Lane

Brea, CA 92621

ORNL: $\mathrm{H}-3$

123 Motorola, Inc.

5005 East McDowell Road

Phoenix, AZ 85008

ORNL: KR-85

124 NASA Space Center

Building 420

Houston, TX 77058

BNL: RSI

$125 \mathrm{NL}$ Erco/NL Industries, Inc. 15702 W. Hardy Road

Suite 240

Houston, TX 77060

ORNL: $\quad$ SR-90

126 National Semiconductor

333 Western Avenue

So. Portland, Maine 04106

ORNL: KR-85

127 National Semiconductor Corp. 5901 S. Calle Santa Cruz Tucson, AZ 85706

ORNL: $\quad K R-85$
128 National Semiconductor Corp.

P.0. Box 700

Santa Clara, CA 95051

ORNL: KR-85

129 Naval Research Laboratory 4555 Overlook Avenue, S.W. Washington, DC 20375

BNL: $\quad N A-22$

130 NeoRx Corporation

410 West Harrison Road

Seattle, WA 98119

LANL: BR-77

ORNL: CSI

131 Neutron Products, Inc.

P. 0. Box 68

Mt. Ephriam Road

Dickerson, MD 20753

INEL: $\quad$ CO-60

ORNL: $\quad$ CO-60, PM-147, RSI

132 New York State Department of Health

Division of Labs and Res.

Empire State Plaza

Albany, NY 12201

BNL: RSI

133 New York University Medical Center Long Meadow Road Tuxedo, NY 10987

BNL: $\quad$ CS -137 
134 New York University Medical Center

P.0. Box 1901

Murray Hill Station

New York, NY 10016

ORNL: MCP

135 North Carolina Memoria] Hospital

$101 \mathrm{E}$. Weaver Street

Carrboro, NC 27510

ORNL: $\quad X E-127$

136 North Carolina State University 214 Clark Labs, Box 7108

Raleigh, NC 27695

LANL: $V-48$

ORNL: CSI

137 North Shore University Hospital

Dept. of Nuclear Medicine

300 Community Drive

Manhasset, NY 11030

BNL: I-125, RU-97, XE-123

LANL: GE -68

ORNL: CSI

138 Northwestern Memorial Hospital 259 East Erie Street

Chicago, IL 60611

ORNL: $\quad$ SR-90

139 Nuclear Radiation Oevelopments Oivision of Mark IV Industries Incorporated

2937 Alt Boulevard North

Grand Island, NY 14072

ORNL: AM-241, CAP
140 Oak Ridge Associated Universities

P.0. Box 117

Oak Ridge, TN 37830

ORNL: MCP, Y-90

141 Oak Ridge National Laboratory P.0. Box $X$

Oak Ridge, TN 37831-6015

BNL: RSI

LANL: BI-207, CU-67

ORNL: CE-144, CF-252, CS-137, CSI, EU-152, GD-153, I-129, MCP, MIS, PU-238, RSI, SP, SR-90, TC-99, TP, U-236

142 Ohio State University Medical Center

Doan Hal1, N163

410 West 10th Avenue

Columbus, $\mathrm{OH} 43210$

BNL: I-125, SM-145

143 Pacific Northwest Laboratory Battelle-Northwest

P.0. Box 999

Richland, WA 99352

ORNL: CF-252, MIS, SP, SS

144 Pett Electronics

858 Hanley Industrial Ct.

Brentwood, M0 63144

LANL: GE-68

ORNL: CSI

145 Piezo Technology, Inc.

P.0. Box 7859

Orlando, FL 32854

ORNL： KR-85 
146 Princeton Plasma Physics Lab

Princeton University

P.0. Box 451

Princeton, NJ 08544

ORNL: CF-252, MIS

147 Princeton University James Forrestal Campus

Princeton, NJ 08540

BNL: AR-37, RSI

ORNL: KR-85

148 Production Data, Inc.

P.0. Box 3266

Bakersfield, CA 93385

ORNL: KR-85

149 Purdue University

West Lafayette, IN 47907

LANL: $\quad \mathrm{CU}-67$

ORNL: CSI

150 R/A Services, Inc.

P.0. Box 13327

Odessa, TX 79766

ORNL: $\quad$ KR-85

151 RCA

Raritan, NJ 08869

ORNL： KR-85

152 Radiation Sterilizers, Inc.

46721 Fremont Boulevard

Fremont, CA 94538

ORNL: $\quad$ CO-60, RSI
153 Raytheon Company

350 Ellis Street

Mountain View, CA 94042

ORNL: $\quad K R-85$

154 Reactor Experiments, Inc.

963 Terminal Way

San Carlos, CA 94070

ORNL: TP, U-235

155 Reuter-Stokes, Inc

Edison Park

8499 Darrow Road

Twinsburg, $\mathrm{OH} 44087$

ORNL: CAP, SP, TP, U-234, $\mathrm{U}=235$

156 Rockwell International

Atomics International Division

Rocky Flats Plant

P.0. Box 464

Golden, C0 80401

ORNL: CSI, RSI, SP, U-236

157 Rutgers State University

P.0. Box 1089

Piscataway, NJ 08854

ORNL: $M G-28$

158 SGS Thomson Microelectronics

P.0. Box 115039, M.S. 2330

Carrollton, TX 75006-5039

ORNL: KR-85

159 Safety Light Corporation

4150-A 0ld Berwick Road

Bloomsburg, PA 17815

ORNL: $\mathrm{H}-3$ 
160 Sandia Corporation

P.0. Box 5800

Albuquerque, NM 87115

ORNL: TP, U-235

161 Sandia National Laboratory

Div. 2126, Bldg. 891

1515 Eubank SE

Albuquerque, NM 87123

BNL: NA-22

162 Saunders-Roe Developments, Inc.

87 Sand Pit Road

Danbury, CT 06810

ORNL: $\mathrm{H}-3$

163 Science Applications

International Corp.

3 Choke Cherry Road

Rockville, MD 20850

LANL: $Y-88$

ORNL: CSI

164 Self-Powered Lighting, Ltd.

8 Westchester Plaza

Elmsford, NY 10523

ORNL: $\mathrm{H}-3$

165 Siemens Gammasonics Health Physics Services

2000 Nuclear Drive

Des Plaines, IL 60018

LANL: GE-68

ORNL: CSI

166 Signetics Corporation

1275 South, 800 East

Orem, UT 84057

ORNL: KR-85
167 Signetics Corporation

811 East Arques Avenue

P.0. Box 3409

Sunnyvale, CA 94088-3409

ORNL: KR-85

168 Siliconix, Inc. 2201 Laurelwood Road

Santa Clara, CA 95054

ORNL: KR-85

169 Solid State Testing 56 Middlesex Turnpike Burlington, MA 01803

ORNL: KR-85

170 Source Production and Equipment Co., Inc.

113 Teal Street

St. Rose, LA 70087-9691

ORNL: IR-192

171 Sprague Solid State 3900 Welsh Road Willow Grove, PA 19090

ORNL: $\quad K R-85$

172 St. Louis University Medical School

Radiation Safety Office 3555 Viata

St. Louis, MO 63110

BNL: $1-125$

173 Stanford University P.0. Box 4409

Stanford, CA 94305

ORNL: CSI, TP, U-238 
174 Stanford University Hospital 820 Quarry Road

Palo Alto, CA 94304

LANL: $\quad V-48$

175 State University of New York at Albany

Research Foundation

1400 Washington Avenue

Albany, NY 12222

ORNL: TC -99

176 State University of New York at Stony Brook

Health Sciences Center

Stony Brook, NY 11794

BNL: I-131, RSI

177 State University of New York Health Sciences Center Department of Physiology Room BSB6-28

Brooklyn, NY 11203

BNL: $\quad I-125$

178 State of New Jersey

Dept. of Env. Protection

Div. of Env. Quality

Bureau of Env. Laboratories

Trenton, NJ 08625

ORNL: $S S$

179 Summa Medical Corporation 4272 Balloon Park Road, N.E. Albuquerque, NM 87109

BNL: $\quad R E-188$
180 TRW Inc.

P.0. Box 922

El Segundo, CA 90245

ORNL: KR-85，SS

181 Technical Wireline Service, Inc.

P.0. Box 1973

Bakersfield, CA 93303

ORNL: $\quad K R-85$

182 Teledyne Isotopes

123 Woodland Avenue

Westwood, NJ 07675

ORNL: KR-85

183 Teledyne Isotopes

Nuclear Systems Division 110 W. Timonium Road

Timonium, MD 21093

ORNL: MCP， SR-90

184 Test-er, Inc.

225 Mitchell Court A10

Chesapeake Square

Addison, IL 60101

ORNL: I-129

185 Texas $A$ and M Research Foundation

P.0. Box $\mathrm{H}$

College Station, TX 77843

ORNL: CSI

186 Texas A\&M University

Radiological Safety office

College Station, TX 77843-3261

LANL: $\quad$ CU-67 
187 Texas Instruments Incorporated Seniconductor Group

P.0. Box $225012 \mathrm{M} / \mathrm{S} 6$

Dallas, TX 75265

ORNL: $\quad$ KR-85

188 Texas Nuclear Corporation P.0. Box 9267

Austin, TX 78756

ORNL: PU-238, TP

189 The Applied Radiant Energy Corporation 2432 Lakeside Drive Lynchburg, VA 24501

ORNL: $\quad C O-60$

190 Theragenics Corporation 900 Atlantic Drive Atlanta, GA 30332

ORNL: SS

191 Tracero Corporation 5763 Underwood Road

Pasadena, TX 77507

ORNL: $\quad$ KR-85

192 Trio-Tech International 355 Parkside Drive

San Fernando, CA 91340

ORNL: $\quad K R-85$

193 Tru-Tec Incorporated

P.0. Box 1908

La Porte, TX 77571

ORNL: $\quad K R-85$
194 U.S. Air Force - Brooks Brooks Air Force Base San Antonio, TX 78235

ORNL: MIS

195 U.S. Air Force - Kirtland Kirtland Air Force Base Albuquerque, NM 87117

ORNL: TP, U-238

196 U.S. Air Force - McClellan McClellan Air Force Base McCleltan AFB, CA 95652

ORNL: AM-241, CF-252, MIS

197 U.S. Army

Armed Forces Radiobiology Research Institute Defense Nuclear Agency Bethesda, MD 20014

ORNL: MIS

198 U.S. Bureau of Mines 729 Arapeen Drive Salt Lake City, UT 84108

LANL: $\quad$ AS-73

199 U.S. Department of Agriculture Research Service P.0. Box 53326 New Orleans, LA 70150

ORNL: MG-28, XE-127

200 U.S. Department of Energy New Brunswick Laboratory 9800 South Cass Avenue Argonne, IL 60439

ORNL: AM-243, SP, TP 
201 U.S. Department of Interior

U.S. Bureau of Mines

4800 Forbes Avenue

Pittsburgh, PA 15213

ORNL: CSI

202 U.S. Geological Survey

1020 0'Brien Orive

Menlo Park, CA 94025

ORNL: CSI

203 U.S. Geological Survey

12201 Sunrise Valley Drive

Reston, VA 22092

ORNL: CSI, H-3, RSI, SP, U-235

204 U.S. Geological Survey

345 Middlefield Road

Men lo Park, CA 94025

LANL: $\quad$ AS -73

205 U.S. National Bureau of Standards

Gaithersburg, MD 20899

ORNL: CE-144, CF-252, GD-153, MIS

206 U.S. National Institutes of Health

900 Rockville Pike

Bethesda, MD 20892

LANL: $\quad$ CU-67, $Y-88$

ORNL: CSI, XE-127, Y-90

207 U.S. Navy

Naval Ocean Systems Center

271 Catalina Boulevard

San Diego, CA 92152-5122

ORNL: MIS, TRU-SV
208 Unisys Corporation

310.1 Goddard Space Flight Center

Greenbelt, MD 20771

ORNL: $\quad K R-85$

209 Unisys Corporation

Computer Systems

P.0. Box 64525

St. Paul, MN 55161

ORNL： KR-85

210 University Hospitals

of Cleveland

2074 Abington Road

Cleveland, $\mathrm{OH} 44106$

ORNL: CSI

211 University of Alabama

Medical Center

1919 Seventh Avenue, South

Birmingham, AL 35486

DRNL: CSI

212 University of Alabama 221 Fourteenth Street S. Birmingham, AL 35294

LANL: BR-77

213 University of Arkansas 4301 West Markham Street Little Rock, AR 72205

LANL: CU-67

ORNL: CSI

214 University of California

405 Hilgard Avenue

Los Angeles, CA 90024

ORNL: $M G-28, X E-127$ 
215 University of California 8655 Production Avenue

San Diego, CA 92121

LANL: $\quad$ CU-67

216 University of California Chemistry Department

Davis, CA 95616

LANL: $\quad Y-88$

ORNL: CSI, MG-28

217 University of California Davis Medical Center 2921 Stockton Blvd.

Sacramento, CA 94817

LANL: $\quad \mathrm{Y}-88$

218 University of California San Diego

La Jolla, CA 92093

ORNL： CSI

219 University of California, Davis $4310 \times$ Street

Sacramento, CA 95817

LANL: $\quad$ CU-67

220 University of California, Davis Internal Medicine 2315 Stockton Blvd., Room 4320 Sacramento, CA 95616

ORNL: CSI

221 University of Central Florida 4000 Central Florida Blyd. Orlando, FL 32816
222 University of Chicago $1525 \mathrm{E}$. Hyde Park Bivd. Chicago, IL 60615

ORNL: TP

223 University of Cincinnati Cincinnati, $\mathrm{OH} 45221$

ORNL: TC-9g

224 University of Colorado Campus Box 54

Boulder, C0 80309

ORNL: TC-99

225 University of Florida Radiation Control office 212 Nuclear Science Center Gainesville, FL 32611

BNL: I -125

226 University of Lowell Research Foundation 450 Aiken Street Lowe 11, MA 01854

ORNL: TP

227 University of Michigan 1101 N. University Bldg. Ann Arbor, MI 48109

LANL: GE-68

ORNL: CSI

228 University of North Carolina Chapel Hill, NC 27514

ORNL： SS

LANL: AS -73

ORNL: CSI 
229 University of Pennsylvania 3400 Spruce Street

Philadelphia, PA 19104-6281

LANL: GE-68

ORNL: CSI

230 University of Rochester 400 Elmwood Avenue

Rochester, NY 14642

BNL: RSI

231 University of Texas

6431 Fannin

Houston, TX 77030

LANL: $\quad$ AS -72

232 University of Texas System Cancer Center

M. D. Anderson Cancer Center

P.0. Box 25128

Houston, TX 77030

ORNL: CSI

233 University of Texas at Dallas 5323 Harry Hines BTvd.

Dallas, TX 75235

LANL: CD-109, CU-67

234 University of Texas at Dallas P.0. Box 30365

Dallas, TX 75230

ORNL: CSI, SS

235 University of Virginia

Radiation Safety Officer

RWMF, 124 11th Street, S.W.

Charlottesville, VA 22903

BNL: $\quad P-32$
236 University of Washington

400 Administration Building

Seattle, WA 98195

ORNL: CSI, SP

237 University of Washington

Isotope Receiving office

Room J25A, Health Scjence

Building

Seattle, WA 98195

LANL: $\quad$ GE -68

238 University of Wisconsin 317 N. Randall Avenue Room 9

Madison, WI 53715

LANL: GE-68

239 University of Wisconsin 750 University Avenue

Madison, WI 53706

ORNL: CSI

240 Washington Department of Social and Health Office of Public Health Labs 1610 N. E. 150th Street

Seattle, WA 98155-7224

ORNL: SP, U-236

241 Washington University 724 S. Euclid, Rm. 242

St. Louis, MO 63110

LANL: BR-77, CU-67, GE-68

242 Washington University Linde11 and Skinker

St. Louis, M0 63130

ORNL: CSI 
243 West Valley Nuclear Services Rocky Springs Road

West Valley, NY 14171-0191

BNL: $\quad$ CS -137

244 Western Kentucky University

Bowling Green, KY 42101

ORNL: MIS

245 Westinghouse Advanced Energy Systems

I-70, Exit 258, Gate A

Madison, PA 15663

INEL: $\quad P 0-210$

246 Westinghouse Electric Corp. P.0. Box 284

Elmira, NY 14902

ORNL: CAP, PU-237, TP, U-234, $\mathrm{U}-235$

247 Westinghouse Electric Corp. Research and Development Center Beulah Road, Churchill Borough

Pittsburgh, PA 15235

ORNL: MIS, NP-237, TRU-SV

248 Westinghouse Hanford Company P.0. Box 1970

Richland, WA 99352

ORNL: MIS

249 Westinghouse Idaho

Nuclear Co., Inc.

P.0. Box 4000

Idaho Falls, ID 83403

ORNL: CM-248, TRU-SV
250 Wright State University

7751 Colonel Glenn Highway

Dayton, OH 45431

ORNL: $\quad M G-28$

251 Yale University

20 Ashmun Street

New Haven, CT 06520

ORNL: $M C P$

252 Yankee Atomic Electric Company 1671 Worcester Road

Framingham, MA 01701

ORNL: CSI

$253 \mathrm{Zi} \log$, Incorporated 10460 Bubb Road

Cupertino, CA 95014

ORNL: $\quad$ KR-85

AUSTRALIA

254 Australian National University Research School of Physical Sciences

Supply Office, G.P.0. Box 4

Canberra, A.C.T. 2601

AUSTRALIA

ORNL: SP, U-236

255 Australian Nuclear Science and Technology Org.

Australian Radioisotopes, 81dg. 238

New Illawarra Road

Lucas Heights, Sydney NSW 2234

AUSTRALIA

LANL: BE-7

ORNL: CSI 
256 Embassy of Australia

1601 Massachusetts Avenue, N.W. Washington, DC 20036

AUSTRALIA

ORNL: PU-239, SP

257 Monash University

The Accountant

Wellington Road

Clayton, Victoria 3168

AUSTRALIA

ORNL: I-129

258 Phillips Communication Systems 2 Greenhills Avenue

Moorebank N.S.W. 2170

AUSTRALIA

ORNL: KR-85

\section{BELGIUM}

259 Institut National Des Radioelements

Service des Achats

Zoning Industrieldu Wainage

B-6200, Fleurus

BELGIUM

LANL: GE-68

ORNL: SR-90

260 Joint Research Centre

Central Bureau for Nuclear Measurements

Steenweg op Retie

$2400 \mathrm{Geel}$

BELGIUM

ORNL: TP
261 Katolieke Universiteit Leuven Inst ituut Voor Kern-En

Stralingsfysika

Celestijneniaan $200 \mathrm{D}$

3030 Leuven

BELGIUM

ORNL: I-129, SS

262 Liege University

Cyclotron Center

B4000 Liege

BELGIUM

LANL: $\quad$ GE-68

263 Universit de Liege

Centre de Recherche du Cyclotron

B-4000 Liege

BELGIUM

ORNL： CSI

BRAZIL

264 Brazilian Center for Physical Research

Rio de Janeiro

BRAZIL

ORNL: $\quad C 0-60, Y-90$

\section{CANADA}

265 Ankha Nuclear Industries (Canada) Inc.

\#3, 2816-21 Street N.E.

Calgary, Alberta

CANADA

0RNL: TC-99 
266 Atomic Energy of Canada, Ltd. Chalk River Nuclear Lab. Chalk River, Ontario CANADA

INEL: RSI

ORNL: $H-3$

267 Atomic Energy of Canada, Ltd. Radiochemical Co.

P.0. Box 13500

Kanata, Ontario K2K $1 \times 8$ CANADA

ORNL: CS-137, MCP, SS, XE-127

268 Canadian Tracerlab, Inc.

P.0. Box 1958, Station "M"

Calgary, Alberta

CANADA

ORNL: $\quad$ KR-85

269 Dr. Gati \& Associates Laboratories Ltd.

P.0. Box 563

Winterburn, ATberta TOE 2NO

CANADA

ORNL: $\quad \mathrm{H}-3$

270 Fisheries and Oceans Freshwater Institute 501 University Crescent Winnipeg, Manitoba R3T 2N6 CANADA

LANL: $\quad$ CD -109

271 Government of Canada

Fisheries and Oceans

Freshwater Institute

501 University Crescent

Winnipeg, Manitoba

CANADA

ORNL: CSI
272 McMaster University

Hamilton, Ontario

CANADA

LANL: GE-68

ORNL: CSI, SP, U-236

273 TRIUMF

4004 Westbrook Mall

Vancouver, BC

CANADA

BNL: U-238

274 Toronto General Hospital

101 College Street

Toronto 2, Ontario

CANADA

ORNL: $\quad Y-90$

275 Universite Du Quebec A Montrea 1

P.0. Box 8888, Branch "A" Montreal (Quebec) H3C 3P8 CANADA

ORNL: $\quad$ TC-99

276 University of British Columbia Accounts Payable 2075 Westbrook Mall Vancouver British Columbia V6T 121 CANADA

ORNL: CSI, MG -28

277 University of Saskatchewan Accelerator Laboratory Saskatoon

CANADA

BNL: $\quad$ CM-244 
DENMARK

278 Isotope Pharmacy, The 378 Frederikssundsvej DK-2700 Bronshoj

DENMARK

ORNL: $\quad$ CS -137

279 Molsgaard Medical A/S Rungstedvej 13 DK-2970 Horsholm DENMARK

ORNL: CS-137, MCP

280 Riso National Laboratory Isotope Division DK-4000 Roskilde

DENMARK

LANL: $\quad V-48$

ORNL: CSI

FINLAND

281 Turku University Central Hospital

Department of Nuclear Medicine SF-20520 Turku

FINLAND

LANL: GE-68

ORNL: CSI

FRANCE

282 CERMEP

59 Bd Pinel

69003 Lyon

FRANCE

ORNL: CSI
283 Centre De Medicine Nucleaire 28 Ave. Du Doyen Jean Lepine 69394 Lyon Cedex 3. M690017 S11

FRANCE

LANL: GE-68

284 Commissariat A L'Energie Atomique

C/0 Embassy of France/Nuclear Office 4101 Reservoir Road N.W. Washington, DC 20007-2180 FRANCE

ORNL: CF-252, MIS

285 Compagnie Oris Industrie Societe Gondrand Roissy-CDC Airport FRANCE

LANL: $\quad$ GE -68

ORNL: CSI

GERMANY (FEDERAL REPUBLIC OF)

286 Federal Health Office

Ingolstaedter Landstr. 1

D-8042 Neuherberg

GERMANY (FEDERAL REPUBLIC OF)

ORNL: AM-243, CM-248, SP, TH-230, TP, TRU-SV, $\mathrm{U}-236$

287 Institut fur Strahlenphysik der Universitat Stuttgart Al lmandring 3 D-7000 Stuttgart 80 GERMANY (FEDERAL REPUBLIC OF)

ORNL: SS 
288 Kernforschungszentrum

Karlisruhe

Postfach 3640

7500 Karls ruhe

GERMANY (FEDERAL REPUBLIC OF)

ORNL: TC-99

289 Kernkraftwerk Obrigheim GmbH 6951 0brigheim am Neckar

Frankfurt

GERMANY (FEDERAL REPUBLIC OF)

ORNL: SP, U-234

290 0sram GmbH LOG/TRE

Postfach 900620

8000 Munchen 90

GERMANY (FEDERAL REPUBLIC OF)

ORNL: KR-85

291 Ruhr Universitat Bochum

Zentrales Isotopten Laboratory

Universitat Strasse 150

4630 Bochum 1

GERMANY (FEDERAL REPUBLIC OF)

LANL: NA-22

292 Universitat Giessen

6300 Giessen

GERMANY (FEDERAL REPUBLIC OF)

ORNL: SP, U-236

293 Universitat Muchen

Sektion Physik

Technologishes Labor

8046 Garching

An Coulombwall 1

GERMANY (FEDERAL REPUBLIC OF)

LANL: $\quad$ AL -26

ORNL: CSI
ISRAEL

294 Rambam Society

Rambam Medical Center

Haifa 35254

ISRAEL

ORNL: MCP

$\underline{\text { ITALY }}$

295 Fondazione Centro S. Romane 110 Del Monte Tabor

Via 0lgettine, 60-Milano

Codice Fiscale 03064280153

ITALY

ORNL: CSI

296 San Raffaele Institute

Nuclear Medicine

Via 0lgettina 60

20132 Milano

ITALY

LANL: $\quad$ GE- 68

JAPAN

297 Dajel Bussan Co., Ltd.

Yoshizumi Building

No. 16, 2-Chome

Nihonbashi Kayabacho Chuo-Ku

Tokyo

JAPAN

ORNL: $\mathrm{H}-3, \mathrm{SS}$

298 Japan Atomic Energy

Research Institute

2-2, Uchisaiwai-Cho 2-Chome

Chiyoda, Tokyo 100

JAPAN

ORNL: CAP, CM-244, H-3, SS 
299 Japan Radioisotope Association Hon-Komagome 2 Chome 28 Bunkyo-Ku

Tokyo

JAPAN

ORNL: TP

KOREA

300 Hyundai Electronics America 4401 Great America Parkway Santa Clara, CA 95054 KOREA

ORNL: KR-85

301 Modu Corporation 33-10 Queens Boulevard Long Is land City, NY 11101 KOREA

ORNL: $\quad$ TC -99

MEXICO

302 Honeywell, S. A. de C. V. Apartado 1223 col Panamericana C. P. 3 Chihuahua, Chih MEXICO

ORNL: KR-85

\section{NETHERLANDS}

303 Byk Mallinckrodt $\mathrm{Ci}$ B. V. Attention: Mr. L. B. Dapper Westeiduinweg 3

1754 Z. G. Petten

NETHERLANDS

ORNL: $\quad T C-99$
304 Vrije Universiteit Amsterdam NETHERLANDS

ORNL: CSI

\section{PHILIPPINES}

305 Team Pacific Corporation Electronics Avenue

FTI Taguig

Metro Manila

PHILIPPINES

ORNL: KR-85

SOUTH AFRICA

$306 \mathrm{~J}$. A. Ewing and McDonald, Inc.

11 Penn Plaza Suite 2221

New York, NY 10001

SOUTH AFRICA

ORNL: $\quad$ TC -99

\section{SHEDEN}

307 Studsvik Ab Purchasing office 61182 Nykoping SWEDEN

ORNL: I-129

\section{SWITZERLAND}

\author{
308 Eidg. Institut fur \\ Reaktorforschung \\ Wurenlingen \\ SWITZERLAND
}

ORNL: $\quad$ AM-243 
309 MB-Microtec, Inc.

Freiburgstrasse 624

$\mathrm{CH}-3172$ Niederwangen/Berne

SWITZERLAND

ORNL: $H-3$

310 Paul Scherrer Institute (PSI)

Formerly SIN

$\mathrm{CH}-5234$ Villigen

SWITZERLAND

LANL: GE-68

ORNL: CSI, H-3

311 Radium-Chemie Limited

$\mathrm{CH}-9053$ Teufen

SWITZERLAND

ORNL: $H-3$

\section{UNITED KINGDOM}

312 Amersham International Great South West Road East Bedfont, Feltham Middlesex England TW14 8NJ UNITED KINGDOM

LANL: $B E-7, \quad Y-88$

313 Amersham Internationa 1, Ltd. White Lion Road Amersham Buckinghamshire England HP7 9LL UNITED KINGDOM

ORNL: CAP, CE-144, CM-244, CO-60, CSI, GD-153, H-3, KR-85, RSI, TC-99, Y-90

314 Medical Research Council Hammersmith Hospital

Ducane Road

London, W12

UNITED KINGDOM

LANL: $\quad$ GE-68
315 Medical Research Council Radiobiology Unit, Harvell Didcot, OxOn, OX11 ORD UNITED KINGDOM

LANL: $\quad$ CU-67

ORNL: CSI

316 Saunders-Roe Developments, Ltd.

Attn: Chief Accountant

Millington Road

Hayes, Middlesex, England UB3 4NB UNITED KINGDOM

ORNL: $\mathrm{H}-3$

317 Semiconductor Devices, Ltd. 65 Wong Chuk Hang Road Gee Chang Hong Centre 16./F1., Flat D, Aberdeen Hong Kong UNITED KINGDOM

ORNL: $\quad K R-85$

318 Sinotra Company Limited Sui Ying Bidg., 1/F Flat 'F'

1 Yuk Yat Street, Lik Shan Road

Kowloon, Hong Kong UNITED KINGDOM

ORNL： SR-90

319 Surelite Limited

Priors Court

Priors Haw Road

Weldon, Northants NN171J6

England

UNITED KINGDOM

ORNL: $H-3$ 
320 The Open University

Finance Division

Sherwood House

Bletchley, Milton Keynes

Buckinghamshire, England

UNITED KINGDOM

ORNL: SP, TH-229

321 Unipath, Ltd.

Norse Road

Bedford MK41 OQG

UNITED KINGDOM

ORNL: $\quad Y-90$

322 University of London

Royal Post Graduate Medical School

Hammersmith Hospita]

London, England W12 OHS

UNITED KINGDOM

ORNL: CSI 


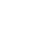




\subsection{LIST OF RADIOISOTOPES PURCHASED CROSS-REFERENCED TO CUSTOMER NUMBERS}

This section contains an alphabetical list of radioisotopes cross-

referenced to customer numbers. This list provides a quick reference of the number of customers purchasing a particular radioisotope. 


\section{ISOTOPES PURCHASED AND CROSS-REFERENCED TO CUSTOMER NUMBERS}

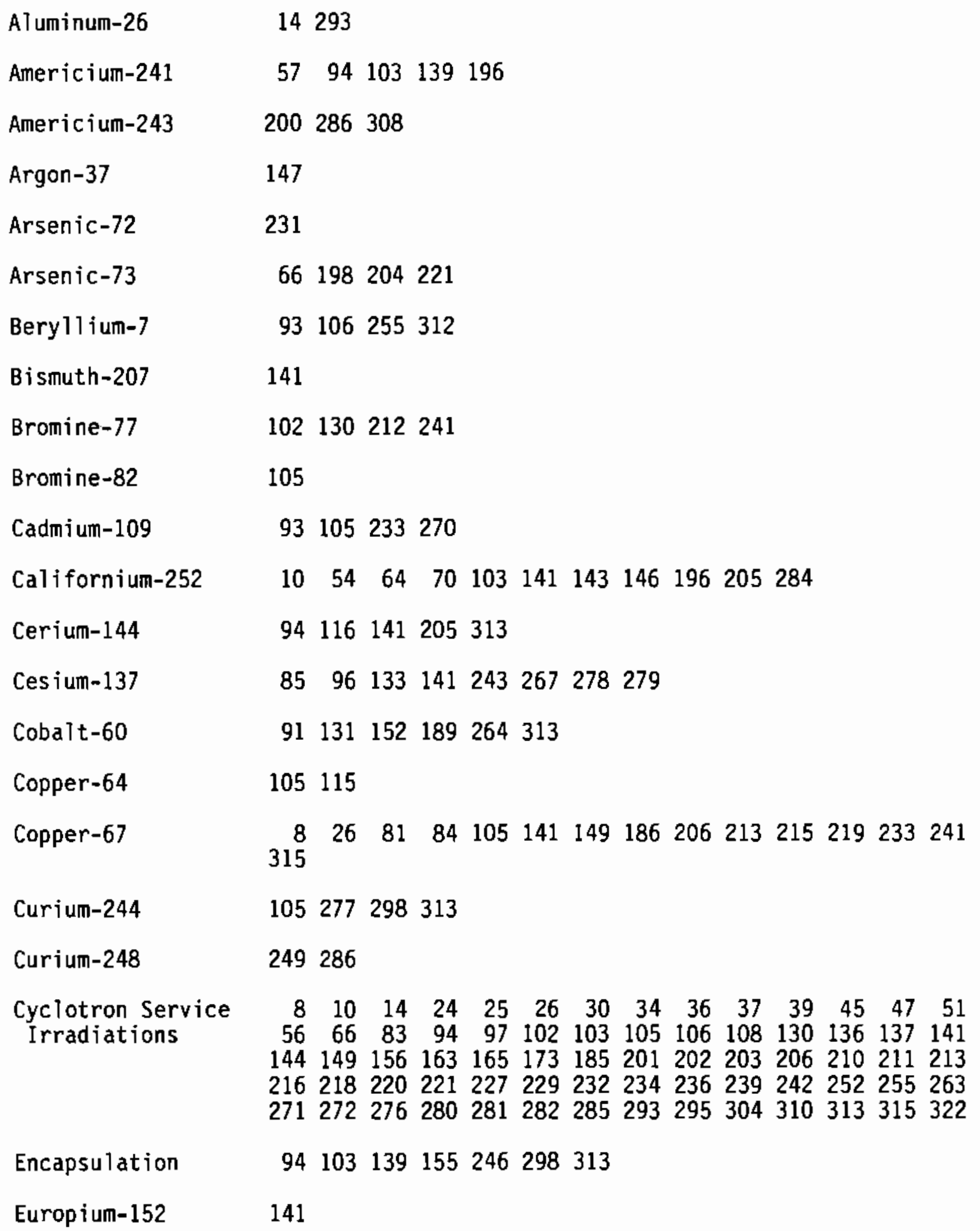




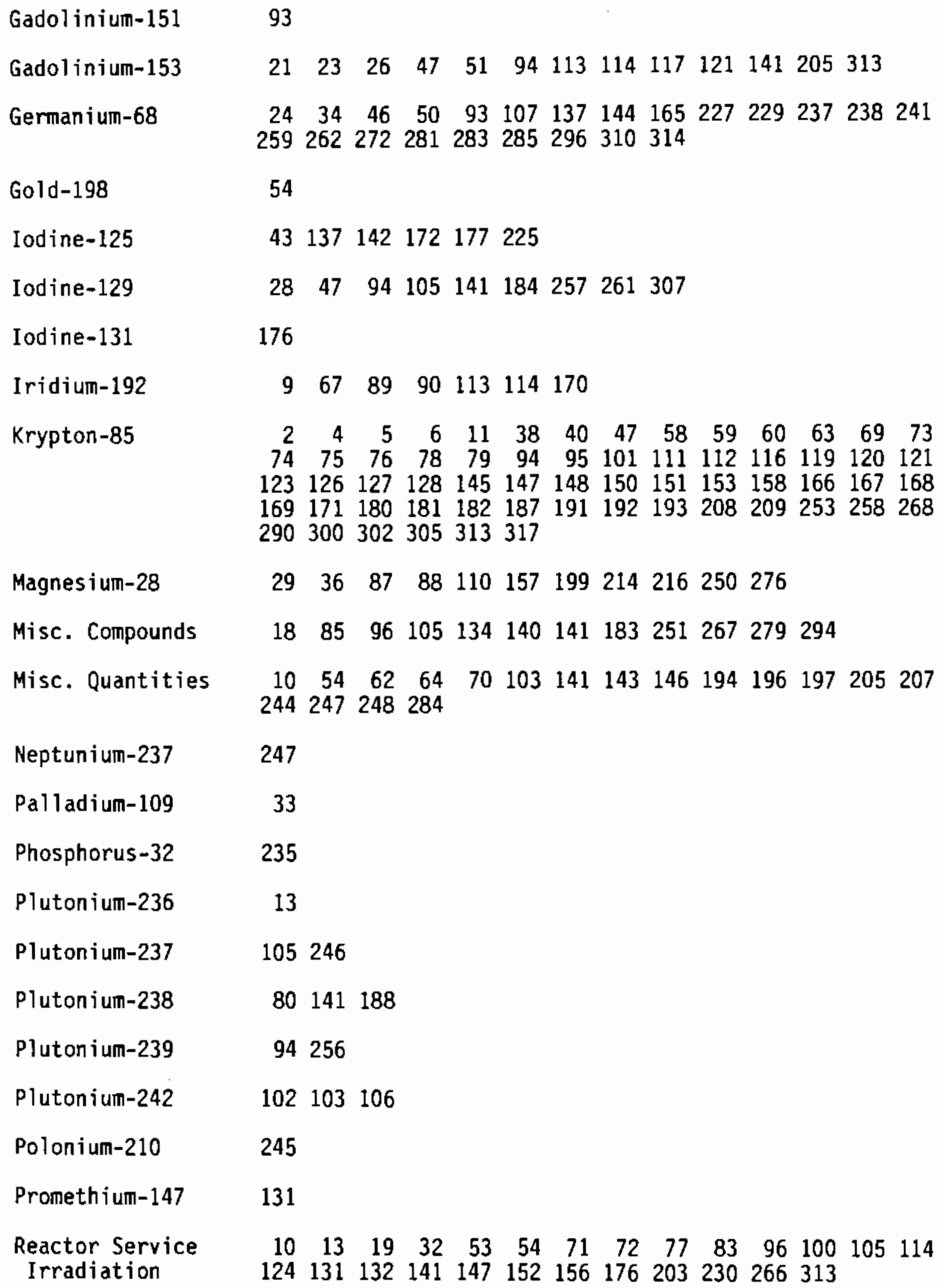




\begin{tabular}{|c|c|}
\hline Rhen i um- 188 & 179 \\
\hline Rhodium-101 & 56 \\
\hline Rubidium-83 & 103 \\
\hline Ruthen ium-106 & 94 \\
\hline Ruthen ium-97 & 137 \\
\hline Samarium-145 & 142 \\
\hline Selenium-72 & 105 \\
\hline Sodium-22 & $\begin{array}{llllll}50 & 104 & 105 & 129 & 161 & 291\end{array}$ \\
\hline Special Processes & $\begin{array}{rrrrrrrrrrrrrr}10 & 12 & 62 & 86 & 94 & 102 & 103 & 105 & 106 & 141 & 143 & 155 & 156 & 200 \\
203 & 236 & 240 & 254 & 256 & 272 & 286 & 289 & 292 & 320 & & & & \end{array}$ \\
\hline Special Services & $\begin{array}{rrrrrrrrrrrrrr}17 & 35 & 39 & 44 & 48 & 65 & 94 & 105 & 114 & 118 & 143 & 178 & 180 & 190 \\
228 & 234 & 261 & 267 & 287 & 297 & 298 & & & & & & & \end{array}$ \\
\hline Strontium-82 & 52102 \\
\hline Strontium-90 & $\begin{array}{lllllllll}47 & 65 & 114 & 125 & 138 & 141 & 183 & 259 & 318\end{array}$ \\
\hline TRU Services & $\begin{array}{llll}11 & 64207247249286\end{array}$ \\
\hline $\begin{array}{l}\text { Target Prepara- } \\
\text { tion }\end{array}$ & $\begin{array}{rrrrrrrrrrrrrr}1 & 10 & 80 & 102 & 103 & 105 & 112 & 141 & 154 & 155 & 160 & 173 & 188 & 195 \\
200 & 222 & 226 & 246 & 260 & 286 & 299 & & & & & & & \end{array}$ \\
\hline Technetium-99 & $\begin{array}{rrrrrrrrrrrrrr}7 & 22 & 31 & 32 & 47 & 49 & 62 & 92 & 98 & 141 & 175 & 223 & 224 & 265 \\
275 & 288 & 301 & 303 & 306 & 313 & & & & & & & & \end{array}$ \\
\hline Thorium-229 & 12320 \\
\hline Thorium-230 & 1062286 \\
\hline Tin-119m & 50 \\
\hline Tritium & $\begin{array}{rrrrrrrrrrrrrr}35 & 47 & 55 & 65 & 68 & 76 & 85 & 99 & 102 & 105 & 122 & 159 & 162 & 164 \\
203 & 266 & 269 & 297 & 298 & 309 & 310 & 311 & 313 & 316 & 319 & & & \end{array}$ \\
\hline Uranium-233 & 10105 \\
\hline Uranium-234 & 10155246289 \\
\hline Uranium-235 & $\begin{array}{lllllllll}1 & 15 & 86 & 112 & 154 & 155 & 160 & 203 & 246\end{array}$ \\
\hline Uranium-236 & 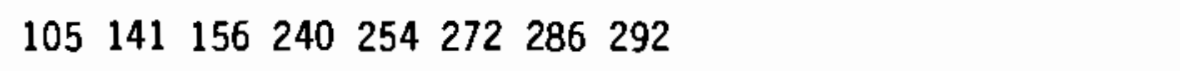 \\
\hline
\end{tabular}




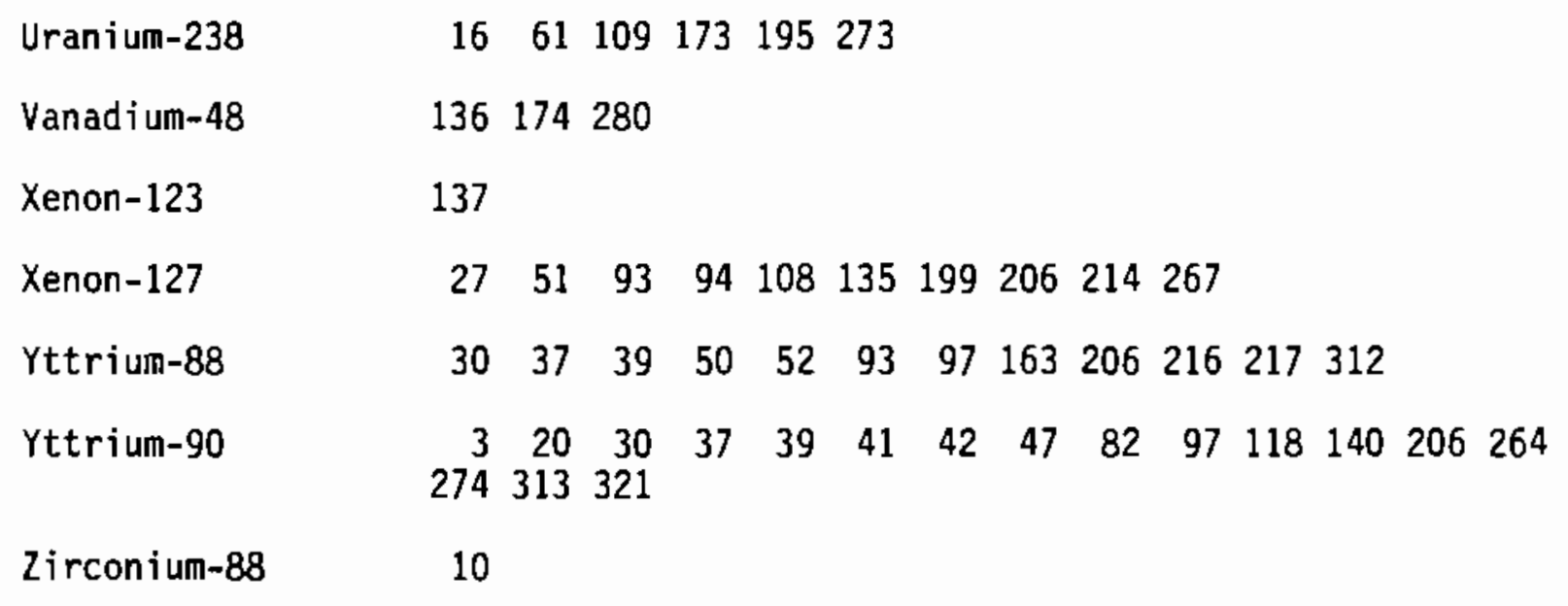





\subsection{GEOGRAPHIC LOCATIONS OF RADIOISOTOPE CUSTOMERS}

This section alphabetically lists states and foreign countries, crossreferencing the customer numbers, which indicate the geographic concentration of radioisotope users. 


\section{GEOGRAPHIC LOCATIONS OF CUSTOMERS BY CUSTOMER NUMBER}

DOMESTIC

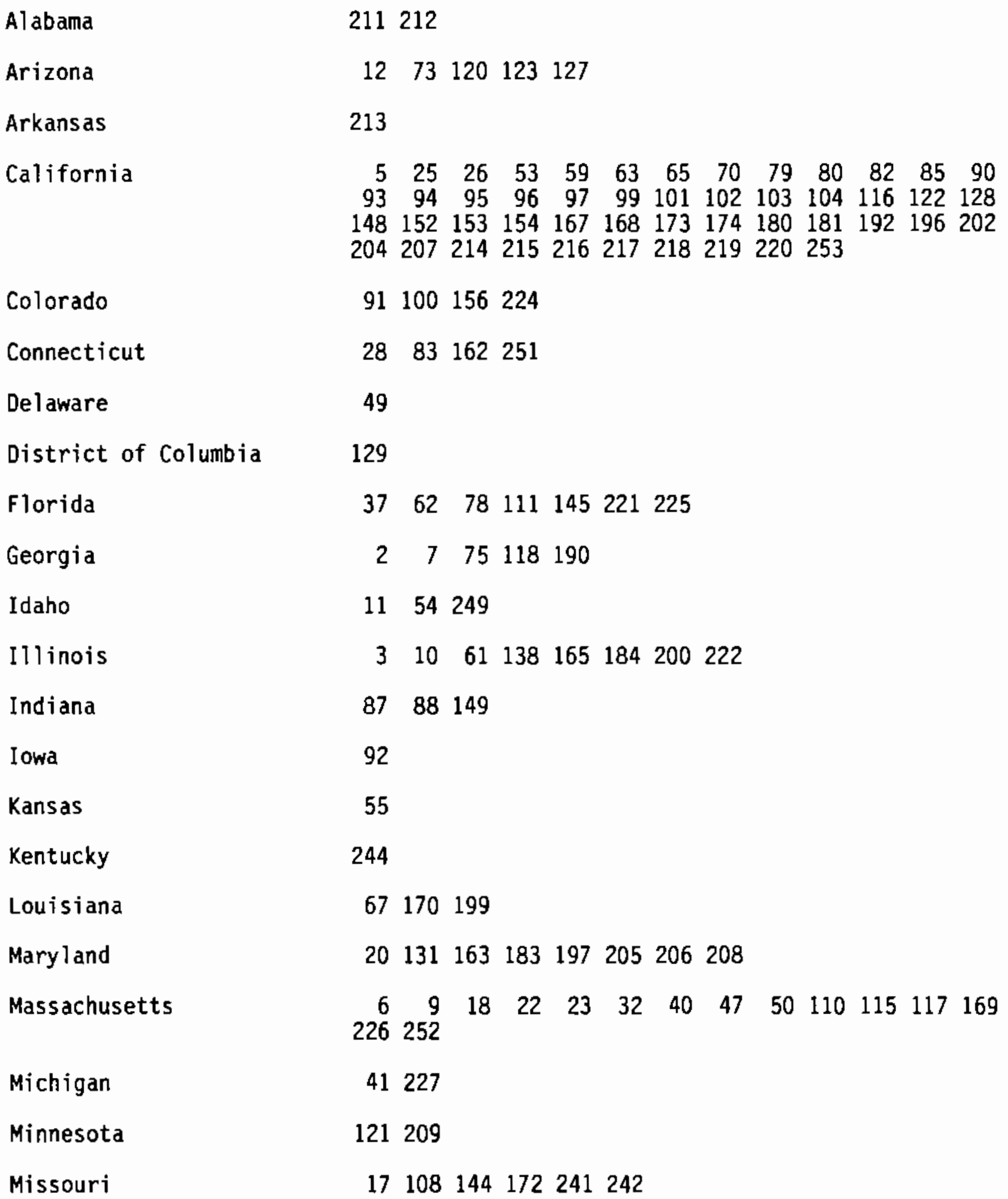




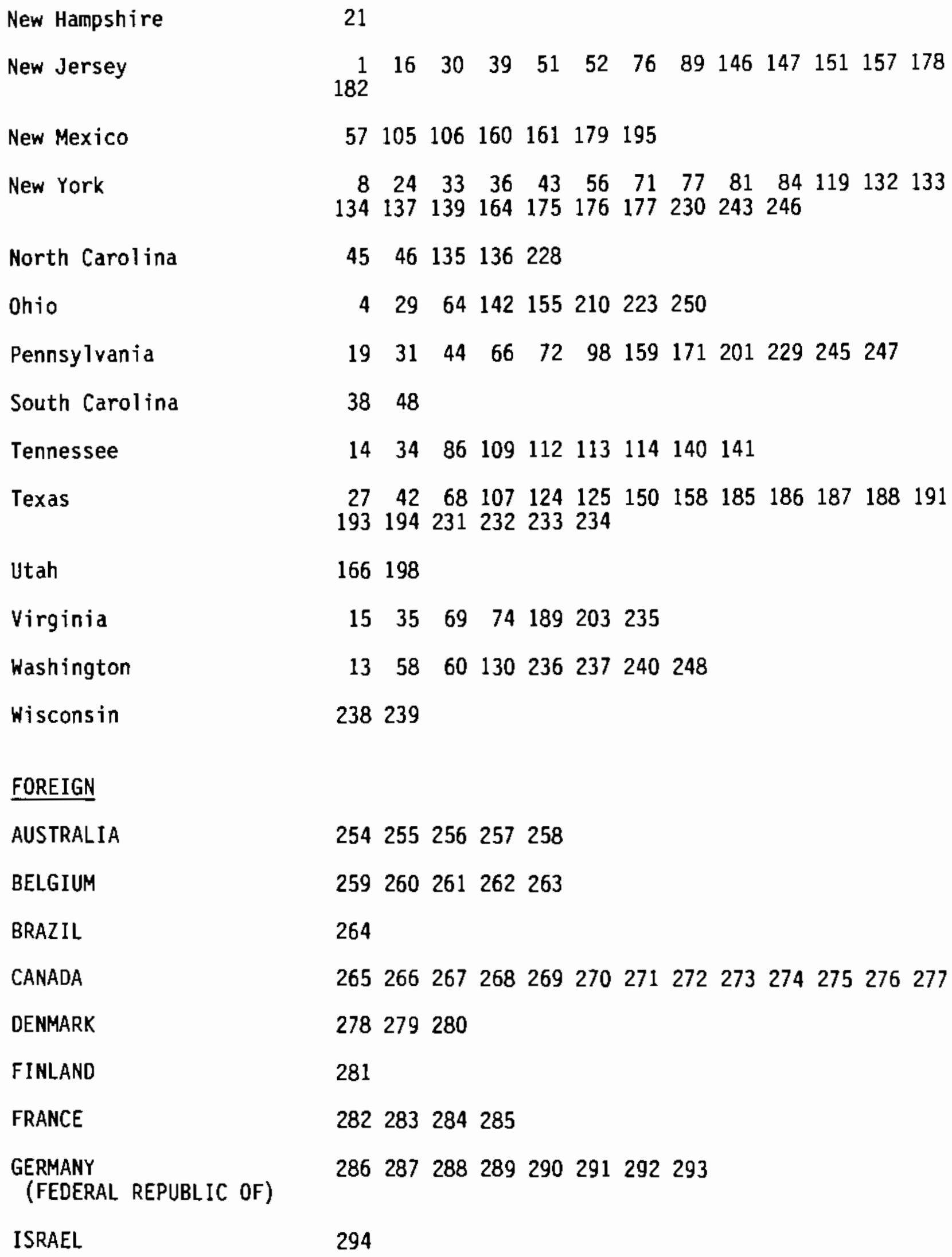


ITALY

JAPAN

KOREA

MEXICO

NETHERLANDS

PHILIPPINES

SOUTH AFRICA

SWEDEN

SWI TZERLAND

UNITED KINGDOM
295296

297298299

300301

302

303304

305

306

307

308309310311

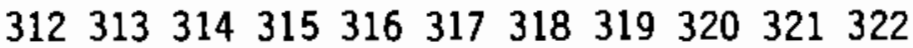




\subsection{RADIOISOTOPE SALES AND TRANSFERS - FY 1988}

This section sumarizes FY 1988 radioisotope shipment activities of the DOE laboratory facilities mentioned in the first section of the report. Radioisotopes are listed alphabetically with the suppliers' abbreviated names. Data on shipments, quantities, units of measure, and dollar values of each radioisotope are listed under domestic, foreign, and DOE categories. The value of the radioisotopes sold in FY 1988 was $\$ 11.1 \mathrm{million}$. 


\section{RADIOISOTOPE SALES AND TRANSFERS - FY 1988}

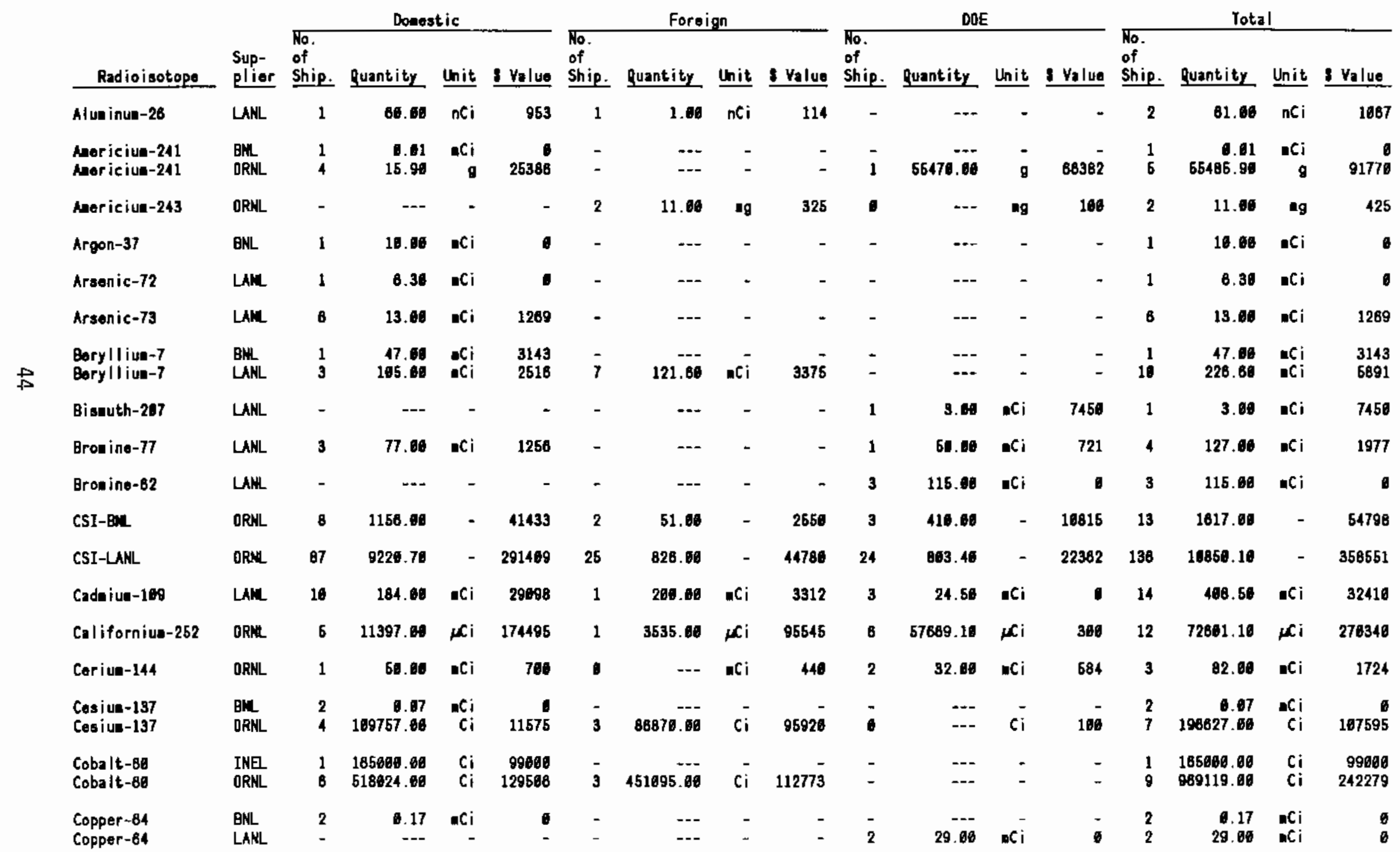




\begin{tabular}{|c|c|c|c|c|c|c|c|c|c|c|c|c|c|c|c|c|c|}
\hline \multirow[b]{2}{*}{ Radioisotope } & \multirow[b]{2}{*}{$\begin{array}{l}\text { Sup } \\
\text { plier }\end{array}$} & \multicolumn{4}{|c|}{ Donestic } & \multicolumn{4}{|c|}{ Foreign } & \multicolumn{4}{|c|}{ DOE } & \multicolumn{4}{|c|}{ Total } \\
\hline & & $\begin{array}{l}\text { No. } \\
\text { of } \\
\text { Ship. }\end{array}$ & Quantity & Unit & \& Value & $\begin{array}{l}\text { Ro. } \\
\text { of } \\
\text { Ship. }\end{array}$ & Quantity & Unit & I Value & $\begin{array}{l}\text { No. } \\
\text { of } \\
\text { Ship. }\end{array}$ & quantity & Unit & I value & $\begin{array}{l}\text { Ko. } \\
\text { of } \\
\text { Ship. }\end{array}$ & Quantity & Unit & I Yaluo \\
\hline Copper-67 & LAM & 46 & 2580.51 & Aci & 6080 & 2 & 25.00 & $n C i$ & 285 & 14 & 1084.60 & C $\mathrm{C}$ & 865 & 81 & 3649.51 & Ci & 6886 \\
\hline $\begin{array}{l}\text { Curiun-214 } \\
\text { Curius-244 }\end{array}$ & $\begin{array}{l}\text { BML } \\
\text { ORNL }\end{array}$ & - & --- & - & - & $\begin{array}{l}1 \\
4\end{array}$ & $\begin{array}{r}169.00 \\
2806.80\end{array}$ & $\begin{array}{l}\mathrm{ACi} \\
\mathrm{Ig}\end{array}$ & 295386 & $i$ & 1.00 & $\dot{g}$ & $10 \overline{-}$ & $\begin{array}{l}1 \\
5\end{array}$ & $\begin{array}{r}186.00 \\
2601.60\end{array}$ & $m g i$ & 295468 \\
\hline Curius-248 & ORN & - & $\ldots$ & - & - & 1 & 6.00 & $=0$ & 725 & 1 & 5006.90 & $\lg$ & 100 & 2 & 5005.80 & $\operatorname{mg}$ & 825 \\
\hline Encapsulation & ORAM & - & $-\cdot$ & - & 665149 & - & --- & - & 360999 & - & -- & - & 42995 & - & --- & - & 1059143 \\
\hline $\begin{array}{l}\text { Europ iun-162 } \\
\text { Europ iuln-152 }\end{array}$ & ORH & - & -- & 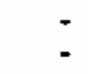 & - & 1 & $\begin{array}{c}0.92 \\
---\end{array}$ & ati & - & $i$ & 2.80 & $m \overline{C i}$ & 185 & $\begin{array}{l}1 \\
1\end{array}$ & $\begin{array}{l}8.92 \\
2.60\end{array}$ & $\begin{array}{l}\mathrm{MCi} \\
\mathrm{MCi}\end{array}$ & $\begin{array}{r}0 \\
185\end{array}$ \\
\hline Gadoliniun-151 & LANL & 1 & 392.00 & $\mathrm{nCi}$ & d & - & $\cdots$ & - & - & - & --- & - & - & 1 & 392.60 & $\mathrm{nCi}$ & $\theta$ \\
\hline $\begin{array}{l}\text { Gadol inius-153 } \\
\text { Gadol inius-163 }\end{array}$ & $\begin{array}{l}\text { INE } \\
\text { ORHL }\end{array}$ & $\begin{array}{r}2 \\
45\end{array}$ & $\begin{array}{l}218.60 \\
141.70\end{array}$ & $\begin{array}{l}\mathrm{Ci} \\
\mathrm{ci}\end{array}$ & $\begin{array}{l}148066 \\
631979\end{array}$ & $\overline{1}$ & 25.06 & $\overline{\mathrm{Ci}}$ & $9435 \overline{-}$ & $\bar{i}$ & 2.0 & $\overline{\mathrm{Ci}}$ & 0139 & ${ }_{50}^{2}$ & $\begin{array}{l}218.68 \\
168.76\end{array}$ & $\begin{array}{l}\mathrm{Ci} \\
\mathrm{Ci}\end{array}$ & $\begin{array}{l}146869 \\
8344 B 8\end{array}$ \\
\hline Goraanius-68 & LANL & 18 & 1036.86 & $\triangle \mathrm{Ci}$ & 62869 & 11 & 385.08 & $\pi \mathrm{Ci}$ & 19116 & 1 & 10.80 & $\triangle \mathrm{Ci}$ & 694 & 30 & 1411.00 & $n C i$ & 72318 \\
\hline $\operatorname{col} d-19 \theta$ & ar & 1 & 1.00 & $\mathrm{DCi}$ & ต & - & --- & - & - & - & $\cdots$ & - & - & 1 & 1.60 & $\mathrm{Ci}$ & $\theta$ \\
\hline Iod ino-123 & Bin & 1 & 4.00 & $A \mathrm{Ci}$ & - & - & -- & - & - & - & --- & - & - & 1 & 1.00 & $\triangle \mathrm{Ci}$ & e \\
\hline Iodine-125 & 日e & 11 & 36.06 & $\mathrm{MCi}$ & I & - & --- & - & - & - & $\cdots$ & - & - & 11 & 38.85 & ${ }^{\mathrm{C}} \mathrm{Ci}$ & 0 \\
\hline Iodine-129 & DRML & 7 & 4614.60 & $m g$ & 29437 & 3 & 384.00 & 19 & 2789 & 3 & 55.86 & $\mathrm{gg}$ & 896 & 13 & 5853.06 & $=8$ & 32833 \\
\hline Iod ine-131 & Ban & 1 & 0.13 & $\mathrm{ACi}$ & - & - & --- & - & - & - & --- & - & - & 1 & 0.13 & $\mathbf{C} \mathrm{i}$ & 0 \\
\hline $\begin{array}{l}\text { Iridiun }-192 \\
\text { Iridium-192 }\end{array}$ & $\begin{array}{l}\text { INA } \\
\text { ORMAK }\end{array}$ & $\begin{array}{l}11 \\
31\end{array}$ & $\begin{array}{l}300622.06 \\
339595.06\end{array}$ & $\begin{array}{l}\mathrm{Ci} \\
\mathrm{Ci}\end{array}$ & $\begin{array}{l}\text { 334868 } \\
461189\end{array}$ & - & -.. & $\overline{-}$ & - & $\overline{8}$ & $809 .--00$ & $\overline{\mathrm{Ci}}$ & 1463 & $\begin{array}{l}11 \\
37\end{array}$ & $\begin{array}{l}306022.60 \\
346198.00\end{array}$ & $\begin{array}{l}\mathrm{Ci} \\
\mathrm{Ci}\end{array}$ & $\begin{array}{l}334099 \\
462592\end{array}$ \\
\hline Krypton-85 & ORIL & 200 & 4608.50 & $\mathrm{Ci}$ & 358771 & 17 & 627.60 & $\mathrm{Ci}$ & 47421 & 2 & 0.40 & $\mathrm{Ci}$ & 533 & 219 & 5233.26 & $\mathrm{Ci}$ & 408725 \\
\hline Krypton-B6E & ORM & 21 & 1132.60 & $\mathrm{Ci}$ & 783939 & 8 & 347.69 & $\mathrm{Ci}$ & 199852 & - & $\cdots$ & - & - & 27 & 1479.06 & $\mathrm{Ci}$ & 993791 \\
\hline Magnes i un-28 & ORAL & 31 & 383.69 & $a c i$ & 21560 & 8 & 7.86 & $\triangle C \mathrm{Ci}$ & 2070 & 4 & 9.06 & $\mathrm{mCi}$ & 1786 & 41 & 399.60 & $m c i$ & 25436 \\
\hline Visc. Conpounds & ORNL & - & --- & - & 4523 & - & -- & - & 1438 & - & --- & - & - & - & -- & - & 5981 \\
\hline Misc. Isotopes & Bin & 45 & --- & - & 2386 & 3 & --- & - & 135 & - & --- & - & - & 48 & --- & - & 2500 \\
\hline Mise. Services & DRM & - & --- & - & 87719 & - & --- & - & 19665 & - & --- & - & 132634 & - & --- & - & 239958 \\
\hline
\end{tabular}




\begin{tabular}{|c|c|c|c|c|c|c|c|c|c|c|c|c|c|c|c|c|c|}
\hline \multirow[b]{2}{*}{ Radioisotopo } & \multirow[b]{2}{*}{$\begin{array}{l}\text { Sup } \\
\text { plier }\end{array}$} & \multicolumn{4}{|c|}{ Domestic } & \multicolumn{4}{|c|}{ Foreign } & \multicolumn{4}{|c|}{ DOE } & \multicolumn{4}{|c|}{ Total } \\
\hline & & $\begin{array}{l}\text { No. } \\
\text { of } \\
\text { Ship. }\end{array}$ & Quantity & Lnit & 5 Value & $\begin{array}{l}\text { No. } \\
\text { of } \\
\text { Ship. }\end{array}$ & Quantity & $\underline{\text { Unit }}$ & I Volue & $\begin{array}{l}\text { No. } \\
\text { of } \\
\text { Ship. }\end{array}$ & Quantity & $\underline{\text { Unit }}$ & S Value & $\begin{array}{l}\text { No. } \\
\text { of } \\
\text { Ship. }\end{array}$ & Quantity & Unit & I value \\
\hline Moptuniun-237H & ORHL & - & -- & g & 287 & - & --- & - & - & - & $\cdots$ & - & - & 由 & -- & $\mathbf{g}$ & 207 \\
\hline Pa | ladiun-189 & Bat & 4 & 1938.06 & aci & - & - & --- & - & - & - & -- & - & - & 4 & 1838.06 & $\mathrm{MCi}$ & E \\
\hline Phosphorus-32 & BlW & 1 & 6.01 & $\mathrm{aCi}$ & $\theta$ & - & -- & - & - & - & -- & - & - & 1 & 0.01 & $\mathrm{MCi}$ & 6 \\
\hline Pluton iua - 237 & ORML & e & -- & $\lg$ & 38060 & - & -- & - & - & 1 & 64.60 & وם & 2863 & 1 & 64.08 & 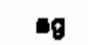 & 48063 \\
\hline Plutoniun-238 & ORM & 2 & 3001.06 & $\mathrm{mg}$ & 6805 & - & --- & - & - & 1 & 180.06 & g & 106 & $\mathbf{3}$ & 3101.06 & $\mathbf{n g}$ & 6965 \\
\hline Plutoni un-239 & DRHL & 1 & 38.08 & $\operatorname{mg}$ & 198 & 1 & 5.80 & $\operatorname{gg}$ & 110 & - & -- & - & - & 2 & 35.60 & $\mathbf{m g}$ & 312 \\
\hline Plutoniun-242 & ORNL & 1 & 16.68 & g & 180 & - & --- & - & - & 2 & 4101.00 & $\boldsymbol{H g}$ & 200 & 3 & 4110.80 & $\bullet$ & 388 \\
\hline Polon iun-210 & INE & 1 & 24.60 & $M C i$ & 888 & - & $\cdots$ & - & - & - & --- & - & - & 1 & 24.06 & $A \mathrm{Ci}$ & 988 \\
\hline $\begin{array}{l}\text { Reactor Sery, Ir. } \\
\text { Reactor Sorv. Ir. }\end{array}$ & $\begin{array}{l}\text { BlHL } \\
\text { ORML }\end{array}$ & $\begin{array}{l}83 \\
13\end{array}$ & 381414.06 & - & $\begin{array}{r}18489 \\
258895\end{array}$ & $\bar{B}$ & 248319.00 & - & 122587 & - & -- & - & 9885 & $\begin{array}{l}63 \\
19\end{array}$ & 627733.00 & - & $\begin{array}{r}16489 \\
392167\end{array}$ \\
\hline Rhen iun-188 & BN & 3 & 1.50 & $\triangle C \mathrm{i}$ & - & - & --- & - & - & - & $\cdots$ & - & - & 3 & 1.68 & $\mathrm{Ai}$ & g \\
\hline Rhodiun-101 & LAHL & 1 & 1.00 & - $\mathrm{Ci}$ & 2282 & - & --- & - & - & - & --- & - & - & 1 & 1.60 & Aci & 2262 \\
\hline Rubidiun-83 & LANL & - & --- & - & - & 1 & 1.38 & $\mathrm{mCi}$ & 163 & - & --- & - & - & 1 & 1.30 & $\mathbf{m C i}$ & 163 \\
\hline Rutheniua-196 & ORNA & 2 & 15.06 & $\mathrm{mCi}$ & 2875 & - & --- & - & - & - & --- & - & - & 2 & 15.00 & $-\mathrm{Ci}$ & 2075 \\
\hline Ruthen iue-97 & B日H & 1 & 23.06 & $\triangle \mathrm{Ci}$ & - & - & --- & - & - & - & $\cdots$ & - & - & 1 & 23.69 & $A C i$ & - \\
\hline 5amariun- 146 & B: & 2 & 8.50 & - $\mathrm{Ci}$ & - & - & --- & - & - & - & $\cdots$ & - & - & 2 & 8.50 & $\mathrm{mCi}$ & 0 \\
\hline Solonius-72 & LAHL & - & --- & - & - & - & --- & - & - & 1 & 0.16 & $\cdot C \mathrm{i}$ & $B$ & 1 & 0.10 & $-\mathrm{Ci}$ & $\theta$ \\
\hline $\begin{array}{l}\text { Sodiun-22 } \\
\text { Sod iun-22 }\end{array}$ & $\begin{array}{l}\text { Bil } \\
\text { LANL }\end{array}$ & $\begin{array}{l}8 \\
3\end{array}$ & $\begin{array}{r}0.08 \\
2208.60\end{array}$ & $\begin{array}{l}\mathrm{MCi} \\
\mathrm{MCi}\end{array}$ & $\begin{array}{r}89683 \\
398\end{array}$ & $\overline{1}$ & 58.-- & $\mathrm{ACi}^{-}$ & 867 & - & --- & $\overline{-}$ & - & $\begin{array}{l}8 \\
4\end{array}$ & $\begin{array}{r}9.80 \\
2258.90\end{array}$ & $\begin{array}{l}n C i \\
m C i\end{array}$ & $\begin{array}{r}6 \\
40556\end{array}$ \\
\hline Special Processes & OPAL & - & --- & - & 3149 & - & --- & - & 16821 & 1 & -- & - & 8623 & 1 & $\cdots$ & - & 26393 \\
\hline Spocial Services & OPAN & 3 & 15.60 & - & 163546 & - & --- & - & 226918 & 1 & 10.00 & - & 34381 & 4 & 25.60 & - & 364839 \\
\hline Stront iun-82 & LAR & 8 & 3345.00 & $\mathrm{mCi}$ & 58535 & - & $\cdots$ & - & - & 2 & 400.60 & $-\mathrm{Ci}$ & 7118 & 10 & 3745.06 & $M C_{i}$ & 65651 \\
\hline Stront iun-90 & ORNL & a & 496216.28 & $\mathrm{Ci}$ & 50123 & 2 & 20.40 & $\mathrm{Ci}$ & 464 & 1 & --- & $\mathrm{Ci}$ & 266 & 9 & 496236.80 & $\mathrm{Ci}$ & 50727 \\
\hline
\end{tabular}




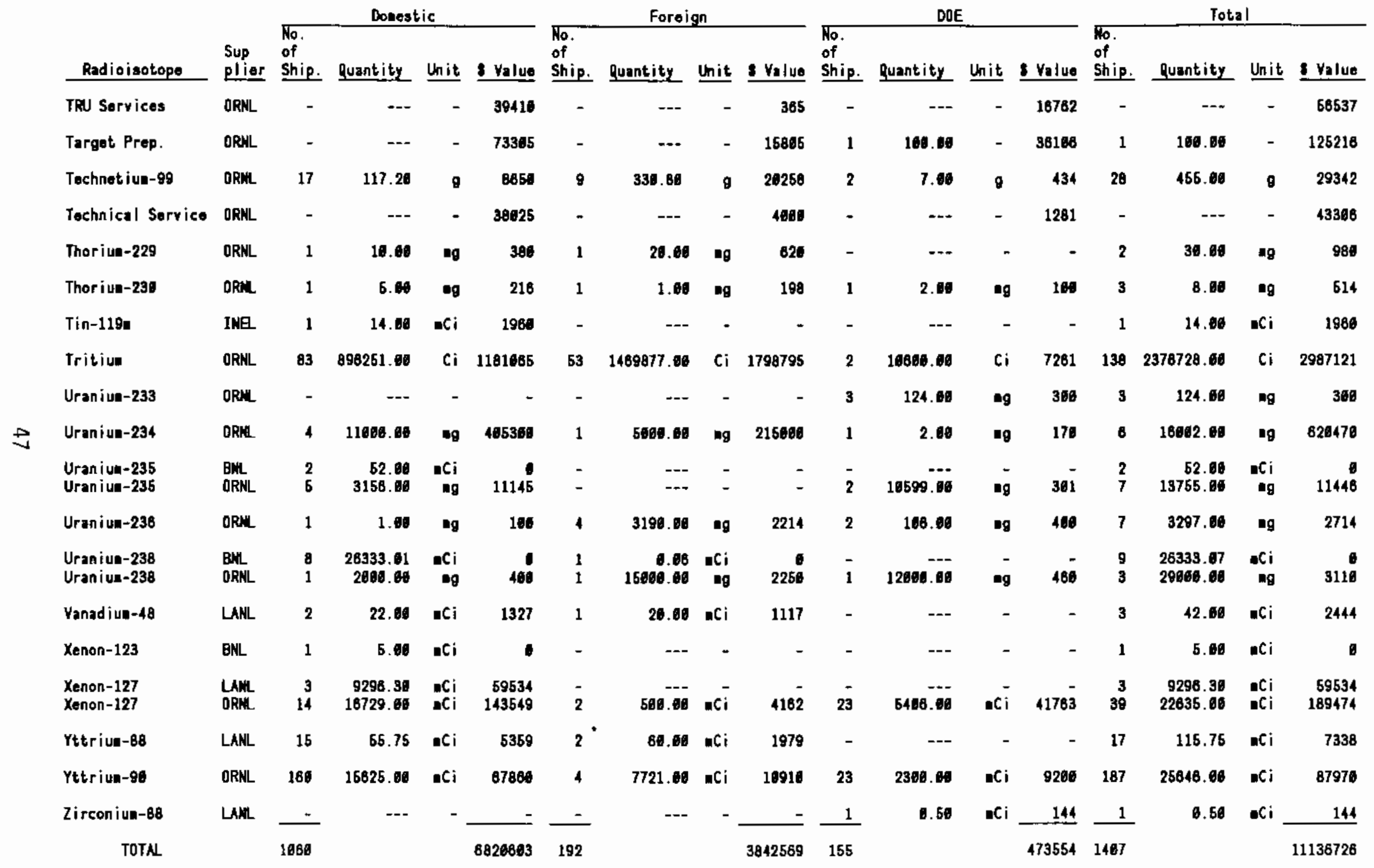



PNL -6934

UC -400

\section{DISTRIBUTION}

No. of

Copies

OFFSITE

10 DOE/Office of Scientific and Technical Information

10 J. N. Maddox

Office of Energy Research, ER-73

U.S. Department of Energy, GTN

Washington, DC 20545

S. N. Ceja, IE-13

U.S. Department of Energy

1000 Independence Ave., S.W.

Washington, DC 20585

Doyle Brown

DOE Oak Ridge Operations office

P.0. Box 2001

Oak Ridge, TN 37831-8610

R. L. Chandler, Director Processing Division

DOE Savannah River Operations office

P.0. Box A

Aiken, SC 29802

R. V. Furstenau

DOE Idaho Operations office

Reactor Facilities and 0ps. Branch

785 DOE Place

Idaho Falls, ID 83402

R. Grandfield

D0E Dayton Area Office

P.0. Box 66

Miamisburg, OH 45343-0066

R. K. Heusser, DP-32

U.S. Department of Energy

Washington, DC 20545
No. of

Copies

Diane Morton

DOE Albuquerque Operations Office/ETWMD

P.0. Box 5400

Albuquerque, NM 87115

L. K. Price

DOE Oak Ridge Operations Office

P.0. Box 2001

Oak Ridge, TN 37831-8610

H. L. Adair

Oak Ridge National Laboratory

P.0. Box 2008

Building 3047, MS-6019

Oak Ridge, TN 37831-6019

J. E. Brasier

Idaho National Engineering Laboratory

EG\&G Idaho, Inc.

P.0. Box 1625

TRA-628, MS 7101

Idaho Falls, ID 83415

2 R. L. Cline

Oak Ridge National Laboratory

P.0. Box 2008

Building 3037, MS-6015

Oak Ridge, TN 37831-6015

D. M. Collins

U.S. Nuclear Regulatory

Commission, Region II

101 Marietta Street

Suite 2900

At lanta, GA 30323

K. S. Dahms

Brookhaven National Laboratory

Associated Universities, Inc.

Isotopes \& Special Materials Group

Upton, Long Island, NY 11973 
No. of

Copies

T. A. Danner

Oak Ridge National Laboratory

Isotope Distribution Office

P.0. Box 2008

Oak Ridge，TN 37831

K. Flayler

EG\&G Mound Applied Technologies

Stable Isotope Sales

P.0. Box 3000

Miamisburg, $\mathrm{OH} \quad 45343-0987$

R. Genung

Oak Ridge National Laboratory

Chemical Technology

P.0. Box 2008

Oak Ridge, TN 37831-6228

J. E. Gindler, Assistant

Director, Chemistry Division

Argonne National Laboratory

9700 South Cass Avenue

Argonne, IL 60439

D. R. Hamilton

DTD/OTA/CDRH

Food and Drug Administration

1901 Chapman Avenue

Mailstop HFZ-240

Rockville, MD 20857

D. R. Hopkins

U.S. Nuclear Regulatory Comnission

Washington, DC 20555

L. F. Mausner

Brookhaven National Laboratory

Associated Universities, Inc.

Upton, Long Is land, NY 11973

J. T. Parks

Idaho National Engineering Lab

EG\&G Idaho, Inc.

P.0. Box 1625, MS 7101

1daho Falls, ID 83415
No. of

Copies

Lisa Schneider

Medical Radioisotopes Research Program

Los Alamos National Laboratory

Group INC-11, Ma 11 Stop J514

Los Alamos, NM 87545

John R. White

U.S. Nuclear Regulatory Commission

475 Allendale Road

King of Prussia, PA 19406

W. H. Smith

EG\&G Mound Applied Technologies

P.0. Box 3000

Miami sburg, $\mathrm{OH} \quad$ 45343-0987

U.S. Nuclear Regulatory Commission

Library

Washington, DC 20555

R. E. Vallee

EG\&G Mound Applied Technologies

P.0. Box 3000

Mianisburg, OH 45343-0987

\section{ONSITE}

2 DOE Richland Operations office

R. A. Almquist

E. C. Norman

8 Westinghouse Hanford Company

S. D. Atkin $\quad \mathrm{HO}-36$

H. R. Brager HO-36

W. R. Brooksher L4-01

L. L. Gilpin L5-05

R. W. Powell L5-55

R. E. Schenter H0-36

A. E. Waltar L5-04

B. D. Zimmerman L5-31 
No. of

Copies

38 Pacific Northwest Laboratory

M. K. Altenhofen

W. J. Bair

L. M. Buel

D. A. Dickman

J. R. Houston

J. H. Jarrett

J. R. Johnson

W. E. Kennedy, Jr.

D. A. Lamar

J. A. Mahaffey

R. W. McKee

R. A. Peloquin (2)

K. L. Swinth

W. L. Templeton

G. L. Tingey

N. C. Van Houten (15)

E. J. Wheelwright

Publishing Coordination

Technical Report Files (5)

Distr.3 
